\title{
Whole-genome microarray analysis and functional characterization reveal distinct gene expression profiles and patterns in two mouse models of ileal inflammation
}

Leela Rani Avula', Dries Knapen ${ }^{2,4}$, Roeland Buckinx ${ }^{1}$ Lucia Vergauwen², Dirk Adriaensen ${ }^{1}$, Luc Van Nassauww ${ }^{1,3}$ and Jean-Pierre Timmermans ${ }^{1 *}$

\begin{abstract}
Background: Although a number of intestinal inflammatory conditions pertain to the ileum, whole-genome gene expression analyses in animal models of ileal inflammation are lacking to date. Therefore, we aimed to identify and characterize alterations in gene expression in the acutely inflamed ileum of two murine models of intestinal inflammation, namely intestinal schistosomiasis and TNBS-induced ileitis, compared to healthy controls. To this end, we used whole-genome microarrays, followed by bioinformatics analyses to detect over-represented Kyoto Encyclopedia of Genes and Genomes pathways and Gene Ontology categories.

Results: Following screening of almost all known mouse genes and transcripts represented on the array, intestinal schistosomiasis and TNBS-induced ileitis yielded 207 and 1417 differentially expressed genes, respectively, with only 30 overlapping concordantly changed genes. Functional category groups consisting of complement and coagulation cascades, extracellular matrix (ECM)-receptor interaction, Fc epsilon receptor I signaling pathways and protein activation cascade, cell adhesion categories were over-represented in the differential gene list of intestinal schistosomiasis. Antigen processing and presentation, cell adhesion molecules, ABC transporters, Toll-like receptor signaling pathways and response to chemical stimulus categories were over-represented in the differential gene list of TNBS-induced ileitis. Although cytokine-cytokine receptor interaction, intestinal immune network for IgA production, focal adhesion pathways and immune, inflammatory and defense response categories were over-represented in the differential gene lists of both inflammation models, the vast majority of the associated genes and changes were unique to each model.

Conclusions: This study characterized two models of ileal inflammation at a whole-genome level and outlined distinct gene expression profiles and patterns in the two models. The results indicate that intestinal schistosomiasis involves Th2 responses, complement activation, protein activation and enhanced ECM turnover, while TNBS-induced ileitis involves Th17 responses, defective antigen processing and presentation and altered Toll-like receptor-mediated responses. Signs of an impaired epithelial barrier are apparent in both inflammation models. Furthermore, the comprehensive differential gene list and functional groups provided by this study constitute an interesting starting point to explore new targets and extended functional networks dealing with small bowel inflammation.
\end{abstract}

Keywords: Gene expression, Intestinal inflammation, lleum, Murine models, Intestinal schistosomiasis, TNBS-induced ileitis, Whole-genome microarrays, Pathways, Over-representation analysis

\footnotetext{
* Correspondence: jean-pierre.timmermans@ua.ac.be

'Department of Veterinary Sciences, Laboratory of Cell Biology and Histology, University of Antwerp, Groenenborgerlaan 171, Antwerp B-2020, Belgium

Full list of author information is available at the end of the article
} 


\section{Background}

Studies in humans and animal models of intestinal inflammation have greatly improved our understanding of some of the underlying dysregulated immune mechanisms, which in turn has promoted the development of new therapeutic strategies. Some of these studies, which were based on whole-genome microarrays for the identification of altered gene expression and transcript regulation at the whole-genome level, have been instrumental in characterizing inflammatory conditions at the molecular genetic level, in obtaining a global picture of the underlying functional processes, as well as in identifying many of the key molecules involved. However, to date, most of these studies involving whole-genome gene expression assays in humans and in animal models of intestinal inflammation have generally targeted the large intestine rather than the small intestine, simply because of tissue accessibility and because of the fact that the majority of the frequently used animal models of both acute and chronic intestinal inflammation involve the large intestine rather than the small intestine [1-5]. As a result, only little is known about the molecular genetic changes underlying small bowel inflammation at the whole-genome level [6]. Nonetheless, it is widely known that a number of major intestinal inflammatory conditions pertain to the ileum, which contains the most complex part of the gut-associated lymphoid tissue, the Peyer's patches, and also harbors a very high luminal concentration of microorganisms [7]. For instance, human Crohn's disease (CD) is most frequently localized in the terminal ileum [7], and it also becomes increasingly evident that human ulcerative colitis (UC) might also involve the terminal ileum due to further extension of the inflammatory process either by backwash from the colon or by other factors [8,9]. A few murine models of small bowel inflammation bearing resemblance to $C D$ have provided vital information on some of the mechanisms underlying small bowel inflammation, but, to the best of our knowledge, whole-genome gene expression studies in the full-thickness ileum have been performed neither in these animal models, nor in other animal models involving any other type of small bowel inflammation to date [10-13].

Studies in mouse revealed that gene expression patterns vary between the anatomically defined regions of the gastrointestinal (GI) tract, such as the stomach, small intestine and large intestine [14]. Moreover, a study based on gene expression in humans suggests that immune regulation is different in ileal and colonic inflammation, which might have important consequences for therapeutic intervention [15]. Consequently, gene expression data on large bowel inflammation cannot be completely extrapolated to other regions of the GI tract, and as such a detailed analysis of the genes involved in small intestinal inflammation is highly desirable. These findings, along with the above-mentioned lack of whole-genome gene expression studies in full-thickness ileum in animal models of intestinal inflammation, stress the urgent need to elaborate on the molecular genetic changes underlying ileal inflammation in view of developing better therapeutic strategies. Therefore, the objective of this whole-genome microarray study was to identify and characterize gene expression changes in the acutely inflamed ileum of two diverse murine models of intestinal inflammation, namely, Schistosoma mansoni-induced intestinal schistosomiasis, which is a non-inflammatory bowel disease model and 2,4,6-trinitrobenzene sulfonate (TNBS)-induced ileitis, which is an inflammatory bowel disease (IBD) model, compared to healthy controls.

Schistosomiasis is a highly prevalent tropical disease caused by the blood-dwelling trematode of the genus Schistosoma, and, although successfully controlled in many countries, remains a major public health problem with an estimated 200 million infected people worldwide [16]. After infection by larvae of S. mansoni, adult worms reach and reproduce in the mesenteric vessel bed and parasite eggs get entrapped in the portal and mesenteric venous systems. A proportion of these eggs penetrate through the liver and intestinal wall to enter the lumen, thereby generating a granulomatous inflammation and subsequent fibrosis [17-19]. Intestinal schistosomiasis has been widely studied in the context of granulomatous inflammation. Moreover, Schistosomaderived eggs and soluble worm proteins have been shown to alleviate intestinal inflammation in animal models of IBD [20,21]. Animal models of granulomatous inflammation, such as schistosomiasis, permit a detailed analysis of basic immune mechanisms involved in chronic immunoregulation that is also relevant to other species $[22,23]$. Therefore, further study of murine intestinal schistosomiasis at the molecular genetic level will lead to a better understanding of its immunomodulatory and immunoregulatory mechanisms.

TNBS is a haptenating agent that immunogenizes autologous or microbiota proteins in the mucosa after disruption of the intestinal epithelial barrier by ethanol. Intraluminal instillation of TNBS in ethanol is known to generate classical models of intestinal inflammation mimicking some aspects of CD [24]. TNBS is one of the best-known chemical agents used for the induction of colitis and ileitis in animal models [25-27]. TNBSinduced inflammation has been widely studied for its resemblance to $C D$ and is considered an apt model to study the acute local inflammatory response as well as the subsequent established and delayed-type hypersensitivity reactions [26].

The present study reports on altered whole-genome gene expression and the associated functional categories and 
pathways underlying acute ileal inflammation in mouse induced by the two aforementioned etiologic factors, i.e., $S$. mansoni parasite-derived eggs, and a chemical agent, TNBS. Our findings collectively provide a solid basis and a starting point for a better understanding of a number of crucial and novel functional processes underlying ileal inflammation initiated by different causative agents.

\section{Methods}

\section{Animal handling}

All experiments were approved by the ethics committee of the University of Antwerp. Adult female C57BL/6 J mice, purchased from JANVIER (Le Genest St Isle, France), were given a standard pellet diet plus water ad libitum, and were housed in a $12 \mathrm{~h} / 12 \mathrm{~h}$ light/dark cycle at constant temperature $\left(22^{\circ} \mathrm{C}\right)$. The animals were divided into three groups: a healthy control group and two inflamed groups, i.e., a group with S. mansoniinduced intestinal schistosomiasis and a group with TNBS-induced ileitis ( $\mathrm{n}=3$ in each group).

Mice were infected with $S$. mansoni according to the method of Yolles et al. [28]. Briefly, the mice were anesthetized with an intraperitoneal injection of sodium pentobarbital (60 mg kg-1. NEMBUTAL; Sanofi, Brussels, Belgium) followed by an intraperitoneal injection of $1 \mathrm{ml}$ of sterile water containing 130 freshly shed cercariae of a Puerto Rico strain of S. mansoni. The cycle of S. mansoni was maintained by passage through Biomphalaria glabrata snails.

TNBS-ileitis was induced by laparotomy according to a modified procedure of Pontell et al. [29,30]. Briefly, after fasting for $24 \mathrm{~h}$, mice were anesthetized using a mix of medetomidine hydrochloride $\left(0.5 \mathrm{mg} \mathrm{kg} \mathrm{kg}^{-1}\right.$; DOMITOR; Pfizer, New York, NY, USA) and ketamine hydrochloride (50 mg kg-1; ANESKETIN; Eurovet, Bladel, the Netherlands) dissolved in physiological solution and administered intraperitoneally. After having been shaved and disinfected, the lower abdomen was incised and the ileum was exteriorized on sterile gauze. A volume of $0.1 \mathrm{ml}$ of $25 \mathrm{mg} \mathrm{ml}^{-1}$ TNBS (Sigma-Aldrich, St. Louis, MO, USA) dissolved in $25 \%$ ethanol was injected transmurally into the lumen of the ileum approximately $2 \mathrm{~cm}$ proximal to the ileo-cecal junction. The ethanolcarrier is included in the basic protocol and is an essential part of the TNBS-inflammation model since it compromises the epithelial barrier, allowing the access of hapten and luminal contents to subepithelial immune cells, thus initiating the immune response [26]. The laparotomy was sutured in two layers using non-resorbable sutures. Before closing the midline incision, a solution containing marbofloxacine (2 mg kg-1 MARBOCYL; Vetoquino S.A., Lure cedex, France) was injected into the peritoneal cavity. After surgery, animals were maintained in a controlled environment for $24 \mathrm{~h}$.
S. mansoni-infected animals were sacrificed 8 weeks post infection at the acute stage of intestinal schistosomiasis. TNBS-treated animals were sacrificed $24 \mathrm{~h}$ post induction at the acute stage of ileitis. All control and inflamed animals were age-matched at the time of tissue retrieval. Since the handling procedures used to induce inflammation in our study are established methods that are not known to induce any inflammatory changes per se, and also taking the high cost of microarray experiments, as well as the reduction of the number of animals for ethical reasons into consideration, we did not consider it necessary to include any additional controls for the inflammation induction procedures in our microarray study $[29,30]$. Although the impact of the survival surgery protocol in the TNBS-treated group was not controlled for in the healthy control group in our study, other studies using the same established surgery protocol and sham treatment revealed a minimal impact of the procedure in itself on these animals [25,27,29,30]. Therefore, we estimate that the gene-expression data in our study might have had a minimal chance of derivation from the surgery protocol by itself. All animals were euthanized in the morning by cervical dislocation, followed by exsanguination. Full-thickness terminal ileum was dissected out and rinsed with Krebs solution (117 mM NaCl, $5 \mathrm{mM} \mathrm{KCl,} 2.5 \mathrm{mM} \mathrm{CaCl}{ }_{2} \cdot 2 \mathrm{H}_{2} \mathrm{O}$, $1.2 \mathrm{mM} \mathrm{MgSO} 4 \cdot 7 \mathrm{H}_{2} \mathrm{O}, 25 \mathrm{mM} \mathrm{NaHCO} 3,1.2 \mathrm{mM} \mathrm{NaH}_{2-}$ $\mathrm{PO}_{4} \cdot 2 \mathrm{H}_{2} \mathrm{O}$ and $10 \mathrm{mM}$ glucose; $\mathrm{pH}$ 7.4). Pieces of ileum weighing around $100 \mathrm{mg}$ each were snap-frozen in liquid nitrogen and stored at $-80^{\circ} \mathrm{C}$ for RNA extraction. A small part of the ileum taken from the same anatomical location as the samples used for the microarray experiments, was fixed for $2 \mathrm{~h}$ at room temperature in $4 \%$ paraformaldehyde in 0.1 $\mathrm{M}$ phosphate buffer ( $\mathrm{pH} 7.4$ ) and processed for paraffin embedding. Five $\mu \mathrm{m}$-thick paraffin sections were stained with hematoxylin-eosin to verify the degree of inflammation. Two pathologists blinded to the disease status and experimental results evaluated the histological specimens by light microscopy.

\section{RNA extraction}

Total RNA was isolated using the TRIzol ${ }^{\circledR}$ extraction method (Life Technologies, Merelbeke, Belgium). In brief, tissues were ground to a fine powder with mortar and pestle in liquid nitrogen, and the homogenized tissues mixed with TRIzol ${ }^{\circledR}$ were used for total RNA isolation according to the manufacturer's protocol. RNA extracts were purified using DNAse I and RNAse inhibitor (Fermentas, St. Leon-Rot, Germany) followed by phenol-chloroform extraction [31]. RNA integrity and purity were verified by denaturing formaldehyde-agarose gel electrophoresis and measurement of 260/230 nm and 260/280 nm absorbance ratios by ND-1000 spectrophotometry (NanoDrop Technologies, Rockland, DE, 
USA). RNA samples were used only if the ribosomal bands showed no degradation on the gel and the 260/ 280 and 260/230 absorbance ratios were between 1.7 and 2.1. A total of 10 ileal total RNA samples were prepared (3 control samples, $3 \mathrm{~S}$. mansoni-infected samples, 3 TNBS-treated samples and one reference sample containing a pool of equimolar amounts of all control total RNA samples).

\section{Fluorescent cRNA labeling and oligonucleotide microarrays}

The two-colour microarray-based gene expression analysis protocol (version 5.7, http://www.agilent.com) was followed to construct and fluorescently label cRNA from total RNA extracts. Briefly, probes were prepared by converting an aliquot of $1 \mu \mathrm{g}$ total RNA from each sample into labeled cRNA, using reagents from the twocolour spike-in kit (catalogue No. 5188-5279, Agilent Technologies, Diegem, Belgium) and a two-colour Quick amp labeling kit (catalogue No.5190-0444, Agilent Technologies). Total RNA was reverse transcribed into firstand second-strand cDNA, after which first-strand cRNA was constructed using the second-strand cDNA as a template, in the presence of either Cy3-CTP or Cy5CTP. Using this method the target material was simultaneously amplified at least a hundred times. The labeled cRNA probes were purified to ensure removal of unbound Cy dyes using the RNeasy mini spin column kit (catalogue No.74104, Qiagen Benelux B.V., Venlo, the Netherlands). Labeling efficiency was determined at $550 \mathrm{~nm}$ (Cy3) and $650 \mathrm{~nm}$ (Cy5) by ND-1000 spectrophotometry (NanoDrop Technologies).

A custom whole-genome gene expression highdefinition oligo microarray platform, (design ID 021695, catalogue No. G2514F), was created and made publicly available in the Gene Expression Omnibus (GEO) database (GPL14111). This custom microarray 4X44K platform represented almost all known genes in the mouse genome and their resulting transcripts in over 4100060 mer oligonucleotide probe features. Experiments were performed on this platform using a reference design as indicated by Knapen et al. [32]. This design consisted of a total of 11 arrays for three biological replicates of control, S. mansoni-infected and TNBS-treated tissue samples as well as two dye flipped technical replicates of a control and a $S$. mansoni-infected tissue sample (see Gene Expression Omnibus (GEO) series accession number GSE31265). Using the Gene expression hybridization kit (catalogue No. 5188-5242, Agilent Technologies) a hybridization sample volume of $100 \mu \mathrm{l}$ was applied on each array containing a total of $825 \mathrm{ng}$ of test sample hybridized against the complementarily labeled reference cRNA. Subsequently, the microarray slides were assembled in the slide chamber and incubated at $65^{\circ} \mathrm{C}$ for $17 \mathrm{~h}$ in a rotating hybridization chamber (Genetix Ltd, New Milton, Hampshire, UK). Slides were stringently washed with wash buffers and acetonitrile and finally submersed in stabilization and drying solution (Agilent Technologies) to prevent ozone-induced Cy5 degradation. Subsequently, the slides were placed in a nitrogen purge box for temporary storage and scanned immediately.

\section{Microarray analysis}

Slides were scanned in the Genepix Personal confocal 4100A scanner (Axon Instruments, Union City, CA, USA) at a resolution of $5 \mu \mathrm{m}$ and at a wavelength close to $532 \mathrm{~nm}$ and $635 \mathrm{~nm}$ for Cy3 and Cy5 channels, respectively. The photomultiplier tube voltages were adjusted to obtain an overall green/red ratio of one. Images were processed using the GenePix Pro 4.1 software (Axon Instruments) for the identification and quantification of the fluorescent signal intensities of the spots [31]. The resulting files were saved as Genepix results (GPR) files, and were imported into the BioArray Software Environment database (BASE 1.2.12, accessible at http://www.islab.ua.ac.be/base/), a free MIAME platform-based microarray analysis method customized by the Intelligent Systems Laboratory (University of Antwerp, Antwerp, Belgium). Through this database, statistical analysis was performed using the $\mathrm{R}$ package limma (linear models for microarray data). Limma is a moderated $T$-statistic approach that detects differentially expressed genes between test groups, given the natural variance within these test groups, adjusted for multiple testing [31,33-35]. Spots for which red or green $\mathrm{FG}<$ $\mathrm{BG}+2 \mathrm{SD}$ as well as spots that were flagged due to artifacts received a weight of zero before analysis (FG: median foreground intensity, BG: average local background intensity calculated over the full microarray, SD: standard deviation of local background intensities) [36]. The median background signal was subtracted from the median foreground signal. The median intensity data were corrected for background using a normal-exponential convolution model with method "normexp", offset $=50$ using the function backgroundCorrect, and loessnormalized using the function normalizeWithinArrays, after which the data were normalized for betweenarray normalization using vsn (variance stabilization) $[31,37,38]$. The two inflammation models were contrasted against the controls, linear models were fitted to intensity ratios, after which probes were ranked in order of evidence of differential expression using an empirical Bayes method [33]. Probes were considered differentially expressed when the $\mathrm{p}$-value was $<0.05$, adjusted $\mathrm{p}$-value (calculated for multiple testing corrections (MTC) on the obtained p-values by the Benjamini and Hochberg false discovery rate method) was $<0.1$ and $\log _{2} \mathrm{FC}$ 
was $>1$ or $<-1 \quad\left(\log _{2} \mathrm{FC}: \log _{2}\right.$ fold change), resulting in a list of differentially expressed probes for each inflammation model. The microarray data have been deposited in the National Center for Biotechnology Information GEO (NCBI GEO; http://www.ncbi.nlm. nih.gov/geo) and are accessible under the GEO series accession number GSE31265.

\section{Bioinformatics data mining and over-representation analysis}

Growing evidence that certain sets of genes acting in concert contribute to the etiology of disease conditions has led to an increased interest in identifying networks or groups of genes, rather than single genes. Moreover, often subtle changes in gene expression, or sometimes the lack of such changes, can equally contribute significantly to changes in function, and these biologically meaningful changes sometimes go undetected by singlegene analysis $[39,40]$. Therefore, we set out to identify biologically relevant category groups in the two inflammation models of our study by performing a functionbased characterization of the differentially expressed genes. To this end, using the stand-alone version of the GO-Elite tool (http://www.genmapp.org/go_elite) [41], we performed a pathway or functional over-representation analysis of the differentially expressed genes for Kyoto Encyclopedia for Genes and Genomes (KEGG) pathways and GO categories. We chose the KEGG pathway analysis rather than analysis of other pathway databases since KEGG is considered to be one of the biggest available pathway databases compiled from several literature sources, especially for mouse $[42,43]$. Since the standalone version of GO-Elite does not work for an overrepresentation analysis of KEGG pathways, we first downloaded the KEGG gpml archive from the PathVisio website (http://www.pathvisio.org), replaced other pathway flat-file relationships already existing in GO-Elite with that of KEGG, and then were able to perform a functional analysis of KEGG pathways in GO-Elite. The input gene set for our over-representation analysis consisted of the differential gene list corresponding to either of the two inflammation models, while the whole genome was used as the denominator or reference background. We used the GO-elite tool with the default options: significance threshold of z-score $>2$ and at least three genes from the input list in the enriched KEGG pathway or GO category. GOelite generated a full list of KEGG and GO annotations associated with all differentially expressed genes, calculated over-representation statistics and provided a minimal list of relevant pruned annotations (filtered for highest scoring terms along each division or branch of the hierarchy and non redundancy in enriched terms) for each inflammation model. The pruned list included main statistics, such as z-score $>2$ (calculated using the expected value and standard deviation of the number of genes meeting the criterion on a pathway under a hypergeometric distribution), permutation p-value (calculated by selecting the original number of input identifiers at random from the denominator identifiers 2000 times), adjusted permutation p-value (calculated using the Benjamini-Hochberg method), average $\log _{2} \mathrm{FC}$ (for each enriched term based on associated differential genes) and average adjusted p-value (for each enriched term based on associated differential genes) for the KEGG and GO terms [41,44]. From this pruned list, we finally set three strict threshold criteria for significance in our overrepresentation analysis, i.e., a $\mathrm{z}$-score $>2$, at least three genes from the input list in the KEGG pathway or GO category and an adjusted permutation $\mathrm{p}$-value $\leq 0.35[41,44]$. These criteria enabled us to identify highly relevant category groups (KEGG pathways + GO biological process categories) in the two inflammation models of our study. Further, GenMAPP (http://www.genmapp.org/) was used for visualization of the gene expression data in the overrepresented KEGG pathways, [41-45].

In addition to the above-mentioned analyses, we briefly compared the list of differentially expressed genes in the two inflammation models of our study with the list of genes previously known to be associated with IBD to assess the degree of similarity between our inflammation models and IBD.

\section{Results and discussion}

The data presented in this study are derived from the analysis of full-thickness murine ileal tissue during physiological and pathophysiological states - i.e., healthy control and the acute stages of intestinal schistosomiasis and TNBS-induced ileitis, respectively, which allowed us to characterize and compare the two models in an intact heterogeneous ileal tissue system. Whole-genome microarrays containing almost all known mouse genes with their resulting transcripts were screened for differential expression in order to unequivocally disclose the differentially expressed genes in the two models and to identify the key functions involved, in terms of KEGG pathways and biological processes.

\section{Histology-hematoxylin-eosin stainings}

No pathological signs of inflammatory activity were observed in the ileum of control mice (Figure 1a), while the ileum of $S$. mansoni-infected mice was characterized by the presence of granulomas consisting of macrophages, eosinophilic granulocytes and lymphocytes surrounding the entrapped parasite eggs in the submucosa and mucosa, by thickening of the tunica muscularis and by broadening of the intestinal villi (Figure 1b). The diffuse mucosal inflammation consisted of granulocytes and scarce lymphocytes. These observations were 


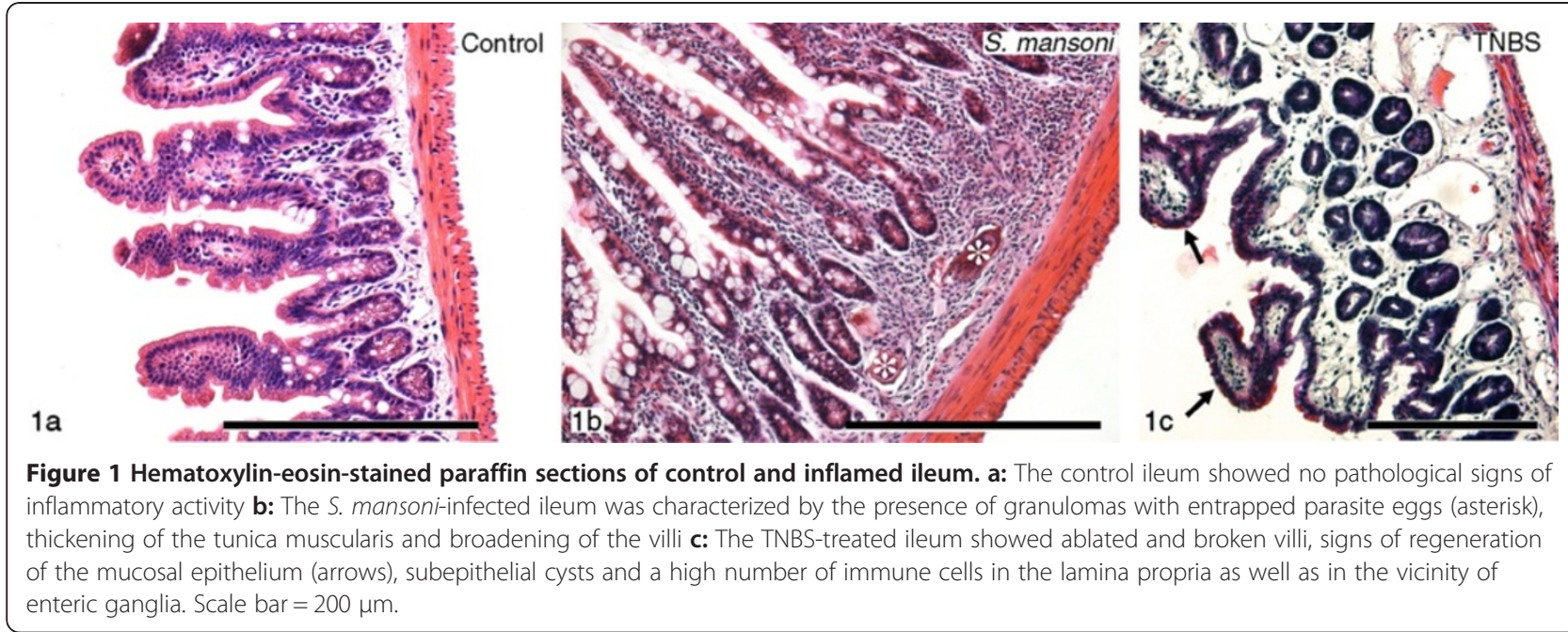

consistent with previous descriptions [17]. Observations in the ileum of TNBS-treated mice were similar to those described in guinea-pig [30]. The villi showed a destructed structure and were ablated, resulting in debris in the lumen, although signs of restoration and regeneration of the mucosal epithelium were observed. Subepithelial cysts and a high number of infiltrated granulocytes, macrophages, along with some lymphocytes within the lamina propria or closely associated with enteric ganglia, were also seen (Figure 1c). As such, all inflamed animals showed obvious acute inflammatory changes, consistent with previous literature data, at the time of tissue retrieval.

As ethanol was used as a vehicle for inducing inflammation in the TNBS-ileitis model, to control for changes induced by ethanol toxicity in itself, the ileum of mice treated with $25 \%$ ethanol alone was also examined by hematoxylin-eosin stainings. Observations in these mice showed no histopathological changes in the ileum after $24 \mathrm{~h}$ (data not shown). These findings are consistent with other studies in mice [27].

\section{Microarray analysis and gene expression profiling}

Statistical limma analysis indicated that 236 and 1685 probes showed differential expression in the S. mansoniinfected and the TNBS-treated ileum, respectively $\left(\log _{2} \mathrm{FC}>1\right.$ or $<-1$; p-value $<0.05$, adjusted p-value $<0.1$, MTC Benjamini-Hochberg). After removal of duplicates, this differentially expressed probe list was found to correspond to a total of 207 unique genes (172 upregulated and 35 downregulated) in the S. mansoni-infected ileum, and to a total of 1417 unique genes (611 upregulated and 806 downregulated) in the TNBS-treated ileum. Analysis of shared gene expression profiles revealed that 48 genes shared differential expression between the two models, i.e., 20 commonly shared upregulated genes, 10 commonly shared downregulated genes and 18 genes that showed opposite changes in the two inflammation models (17 genes upregulated in the S. mansoni-infected ileum and downregulated in the TNBS-treated ileum, and 1 gene downregulated in the S. mansoni-infected ileum and upregulated in the TNBS-treated ileum). Detailed information on all differentially expressed genes is found in the additional files (see Additional file 1 and Additional file 2).

The above findings clearly demonstrate that, in general, the number of genes showing differential expression is higher in the TNBS-treated ileum than in the S. mansoni-infected ileum (approximately 6 times more), implying that TNBS-induced ileitis has a more profound impact on ileal gene expression than intestinal schistosomiasis. Furthermore, the vast majority of differentially expressed genes in the $S$. mansoni-infected ileum were upregulated, while downregulated genes narrowly outnumbered upregulated genes in the TNBS-treated ileum. More interestingly, only a minority (30 genes) of all the differential genes overlapped between the $S$. mansoniinfected ileum and the TNBS-treated ileum in a concordant manner. Together, these findings indicate that the gene expression profiles in the two inflammation models are considerably different, likely owing to the different inflammation-inducing agents.

\section{Gene ontology (GO) and pathway over-representation analysis}

The full list of KEGG and GO annotations in GO-Elite showed that of the whole mouse genome represented on the arrays, 5699 individual genes were contained within the GO-elite KEGG database and 17730 individual genes were contained within the GO-elite GO database, and were used for analysis. Of the 207 unique genes that were differentially expressed in the $S$. mansoni-infected 
ileum, 42 genes could be linked to a KEGG pathway term and 144 genes to a GO term. Of the 1417 unique genes that were differentially expressed in the TNBStreated ileum, 232 genes could be linked to a KEGG pathway term and 1050 genes to a GO term. Additional files 3 and 4 detail all the differentially expressed genes that could be linked to a KEGG pathway and/or GO term in the $S$. mansoni-infected and the TNBS-treated ileum respectively (see Additional files 3 and 4).

The above findings clearly show that although the vast majority of the differentially expressed genes in the two inflammation models of our study could be linked to a GO term and/or a KEGG pathway term, there still remained a significant number of differentially expressed genes that could not be associated with a KEGG pathway term $(\approx 80 \%$ in the $S$. mansoni-infected ileum and $\approx 84 \%$ in the TNBS-treated ileum) or a GO term $(\approx 30 \%$ in the S. mansoni-infected ileum and $\approx 26 \%$ in the TNBStreated ileum).

Based on the above-described threshold criteria for over-representation analysis described in the methods section, nine KEGG pathways were over-represented in the upregulated gene list of the S. mansoni-infected ileum. Only one KEGG pathway was over-represented in the downregulated gene list of the $S$. mansoni-infected ileum. Twenty-six GO biological process categories were over-represented in the upregulated gene list of the $S$. mansoni-infected ileum, and three GO biological process categories were over-represented in the downregulated gene list of the $S$. mansoni-infected ileum. Detailed information on all over-represented KEGG pathways and GO categories in the S. mansoni-infected ileum are found in additional file 5 (see Additional file 5).

Twenty-six KEGG pathways were over-represented in the upregulated gene list of the TNBS-treated ileum. Twenty-eight KEGG pathways were over-represented in the downregulated gene list of the TNBS-treated ileum. Ninety-seven GO biological process categories were over-represented in the upregulated gene list of the TNBS-treated ileum and seventy-one GO biological process categories were over-represented in the downregulated gene list of the TNBS-treated ileum. Detailed information on all over-represented KEGG pathways and GO categories in the TNBS-treated ileum are found in additional file 6 (see Additional file 6).

Identification of functionally meaningful category groups Given the high number of over-represented KEGG and GO terms, we considered it relevant to make a shortlist of the most significant category groups. Based on the high overlap of individual genes observed among some of the high scoring KEGG pathways and GO categories, five functionally meaningful category groups (KEGG pathways + GO categories) were finally obtained in the $S$. mansoni-infected ileum (Additional file 7: Table S1):

1. Cytokine-cytokine receptor interaction pathway and immune and defense response categories;

2. Intestinal immune network for immunoglobulin A (IgA) production pathway and immune response category;

3. Complement and coagulation cascades pathway and protein activation cascade category;

4. Extracellular matrix (ECM)-receptor interaction pathway and cell adhesion category;

5. Fc epsilon RI (FceRI) signaling pathway and regulation of acute inflammatory response category.

Six functionally meaningful category groups were finally obtained in the TNBS-treated ileum (Additional file 8: Table S2):

1. Cytokine-cytokine receptor interaction pathway and inflammatory and immune response categories;

2. Intestinal immune network for IgA production pathway and immune response category;

3. Antigen processing and presentation pathway and antigen processing and presentation of endogenous antigen, of exogenous peptide antigen and of peptide or polysaccharide antigen via major histocompatibility complex (MHC) class II categories;

4. Cell adhesion molecules pathway and cell adhesion category;

5. Toll-like receptor (TLR) signaling pathway and immune and inflammatory response categories;

6. ABC transporters pathway and response to chemical stimulus category.

These functional category groups will be elaborated on below. The category groups pertaining to both inflammation models will be discussed first, followed by those that are relevant only to the $S$. mansoni-infected ileum and then those relevant only to the TNBS-treated ileum. Wherever necessary, in order to group important information, changes pertaining to genes that are not contained in the category group but are of relevance will also be discussed.

\section{Cytokine-cytokine receptor interaction pathway and immune response category group}

(Additional file 1, Additional file 2, Additional file 7: Table S1 and Additional file 8: Table S2) (Figure 2).

In the $S$. mansoni-infected ileum, our findings suggest a predominant presence of polarized Th2 responses during acute intestinal schistosomiasis, and that the cytokine-cytokine receptor interaction pathway-immune 


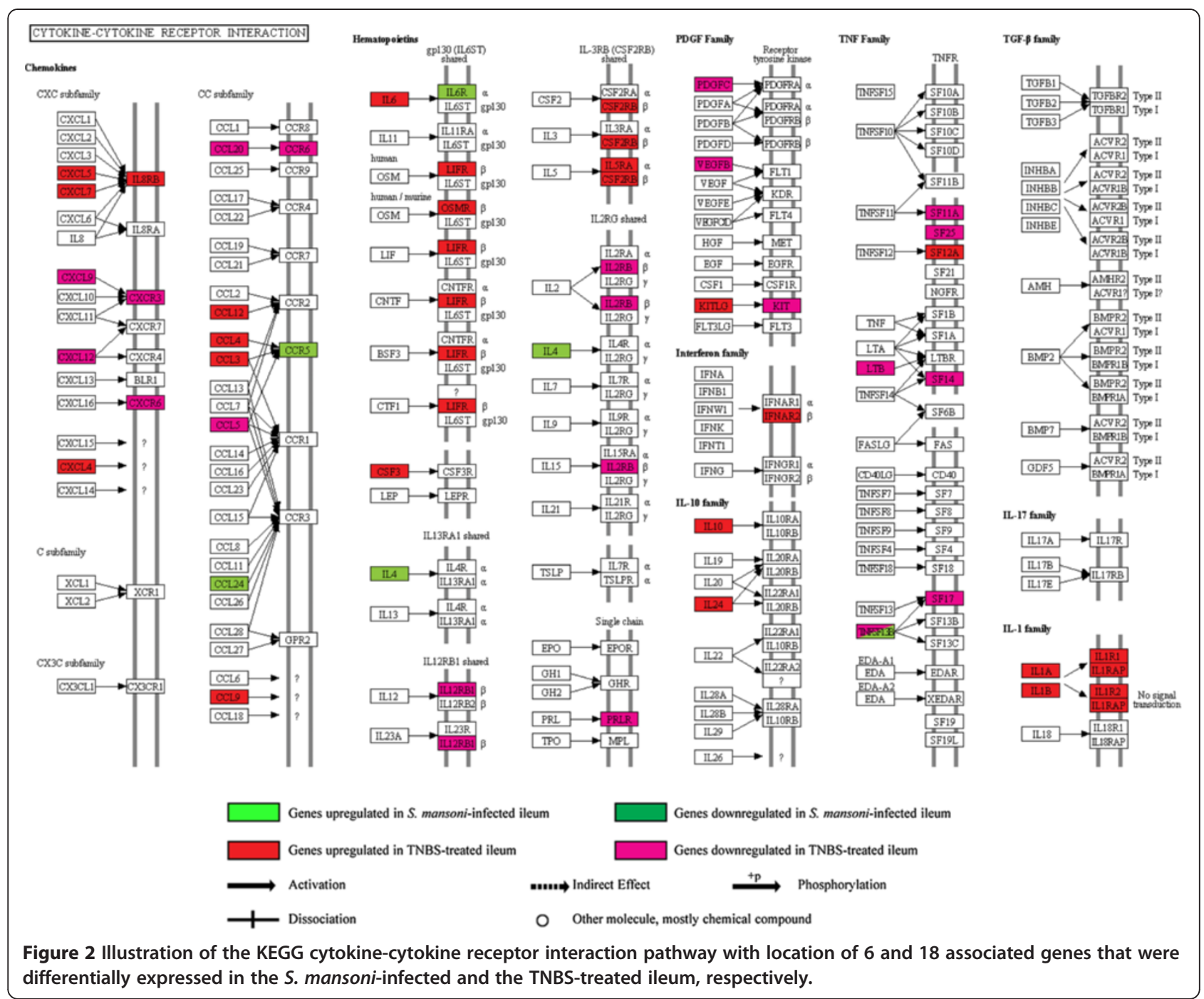

(and defense) response category group is upregulated. These findings are in agreement with previous reports showing that the inflammatory reaction to schistosome eggs in the mouse intestine is predominantly a Th2-type response [46]. The cytokine-cytokine receptor interaction pathway and immune (and defense) response category group shared 6 genes (Ccl24, Ccr5, Il18r1, Il4, Il6ra, Tnfsf13b), all of which showed increased expression. Increased expression of $\mathrm{Il} 4, \mathrm{Ccl} 24$ and Ccr5 is indicative of Th2-type responses [47-49] and IL4 is a hallmark cytokine for Th2-type $\mathrm{T}$ cell responses [47]. Upregulation of Ccl24 (eotaxin-2) is associated with recruitment of immune cells including eosinophils and Th2 cells in the GI mucosa during helminth infection [48]. The increased expression of the Ccr5 found in our study lends further support to literature data showing that CCR5 plays a role in limiting the granulomatous and Th2 responses, thereby reducing severity of experimental schistosomiasis [49]. Furthermore, the increased expression of resistin-like beta (Retnlb/Fizz2) in the S. mansoniinfected ileum supports the notion that the protein encoded by this gene, intestinal goblet cell-derived RELMbeta/FIZZ2, is a Th2 cytokine-induced immune-effector molecule produced in resistance to $S$. mansoni infection [50]. RELM-beta/FIZZ2 has also been associated with the initiation of ileitis in animal models of CD [10]. The highly increased expression of other Th2 cytokine-associated genes, such as chitinase-3-like genes Chi3l3 and Chi3l4 indicate their probable involvement in host/microbial interactions and thereby in the disease pathogenesis in this model [51].

In the TNBS-treated ileum, our findings suggest that proinflammatory responses, Th17 priming and regulatory mechanisms are enhanced during acute TNBS-induced ileitis, and that the cytokine-cytokine receptor interaction pathway-immune (and inflammatory) response category 
group is upregulated under this condition. This particular category group shared 8 genes ( $\mathrm{Cl} 3, \mathrm{Ccl} 4, \mathrm{Ccl}$, $\mathrm{Cxcl5}$, $I l 1 b, I l 6, P f 4, P p b p$ ), all of which showed increased expression. Increased expression of $I l 6$ and $I l 1 b$ and of genes such as the transcription factor Stat3, in the TNBStreated ileum indicate changes that are associated with Th17 cell induction and differentiation [52-54]. Data on other effector cytokines of Th17 cells, such as $I l 17 a$, were not available in our differential gene list, probably resulting from the very low gene expression levels of Th17 effector cytokines. The observation that Ccr6 shows decreased expression in the TNBS-treated ileum, together with the increased expression of $I l 6$, could be indicative of increased Th17 effector responses and decreased Foxp3induced regulatory responses in the TNBS-treated ileum $[55,56]$. Furthermore, some of the selected genes induced in Th17 cells compared to Th0 cells, such as the abovementioned Ccl9 and Tnfrsf12A, showed increased expression in this condition [57]. Taken together, the TNBStreated ileum shows gene profiles indicative of Th17 cell priming. It has been reported earlier that CCL9 is involved in the recruitment of CD11b + dendritic cells (DCs) and that CD11b + DCs preferentially induce Th17 development $[53,58,59]$. Probably in keeping with this finding, is our observation that Ccl9 together with integrin $\alpha \mathrm{M}$ $($ Itgam $/ C d 11 b)$ is increasingly expressed, which points to a role of CD11b + DCs in the TNBS-treated ileum of Th17 cell development. Furthermore, the increased expression of S100A8 and S100A9 genes in this model is most likely related to neutrophil abundance and chemotactic effects related to innate immunity, which in turn might be linked to Th17 cell responses, since neutrophils are considered to be the prime effector cells and inducers of Th17 differentiation $[60,61]$. We further observed a decreased expression of transcription factors T-box 21 (Tbx21/Tbet), as well as of the preferential receptor for Th1 cells, Cxcr3. In the TNBS-treated ileum, these changes suggest inhibition of Th1 cell development in favor of Th17 cell development, whereby Th17 cells further inhibit the Th1 cell development [52,62-64]. About the chemokines and their receptors, increased expression of Cxcl4, Cxcr3, Ccl2-4, Ccl9 point to recruitment of immature DCs, T cells, monocytes or neutrophils [65], while other members of this group ( $\mathrm{Cxcl9}$, Cxcl12, Ccl5, Ccl25 and Ccr6) exhibited a decreased expression. The repressed expression of $\mathrm{Cxcl9}$ is probably related to the downregulated Th1 responses, which again is in agreement with the observation that Th17 cells suppress Th1 cell differentiation [52]. The decreased expression of $\mathrm{Ccl} 25$ is probably associated with the reduced homing of $\mathrm{T}$ cells and IgA + plasma cells to the intestine, which is consistent with suppression of the intestinal immune network for IgA production in this model, as will be discussed later [65]. Another interesting change that we observed in relation to the cytokines in this model, was the increased expression of Il33, a ligand for the IL1 family receptor T1/ST2. IL33 is mainly known as an inducer of Th2 differentiation, but has recently been attributed crucial functions in amplifying mucosal innate rather than acquired immune responses [66,67]. Moving on to regulatory mechanisms, increased expression of the Il1 receptor antagonist Il1rn in this model, in the light of elevated $I l 1 b$ expression, supports the view of host mechanisms for the regulation of the IL1 system, as also observed in IBD [54]. The increased expression of Thbs1 encoding thrombospondin 1 (THBS1) suggests that THBS1 is involved in the clearance of inflammatory cells in this model [68]. The increased expression of the regulatory cytokine $I l 10$, retinol-binding protein $1(R b p 1)$ and retinol dehydrogenase 10 (Rdh10) might be relevant in the light of a recent study demonstrating that during inflammatory conditions, Th17 cells of the regulatory phenotype (rTh17) also expressed high levels of IL10 and that the control of Th17 cells occurs in the small intestine [69]. These data suggest the in vivo presence of immunosuppressive activity and reciprocal regulatory responses in the TNBS-treated ileum, and hence point to the presence of host mechanisms that tune down exaggerated pro-inflammatory Th17 responses, in an attempt to reinforce intestinal homeostasis [52,70]. These findings are in line with previous studies, in which C57BL/6 and other mouse strains were used and which demonstrated that acute TNBS inflammation is self-limiting, with a naturally occurring healing response. This healing response was shown to involve the production of regulatory cytokines, which thereby cause the inflammation to eventually reach near complete remission shortly after the acute stage [21,27].

\section{Intestinal immune network for IgA production pathway and immune response category group}

(Additional file 1, Additional file 2, Additional file 7: Tables S1 and Additional file 8: Table S2).

In the $S$. mansoni-infected ileum, our results demonstrate that the intestinal immune network for IgA production pathway and immune response category group is upregulated, conferring defense against toxins and microorganisms. The category group shared 3 genes (Il4, Itgb7, Tnfsf $13 b$ ), all of which showed increased expression. Using in vitro methods, Crabtree et al. reported the increased immunoglobulin production in S. mansoni infections [71]. Probably related to these observations is the increased expression of the immunoglobulin heavy chain related gene Ighv6-6 in the S. mansoni-infected ileum.

In the TNBS-treated ileum, our findings suggest that protective immune responses, the antibody line of defense and epithelial integrity are affected, leading to insufficient defense against microorganisms. The category group shared 10 genes (Ccl25, Cxcl12, H2- $\mathrm{Aa}, \mathrm{H} 2-$ 
$A b 1, H 2-D M a, H 2-D M b 1, H 2-D M b 2, H 2-E b 1, H 2-O b$, Tnfsf13b), all of which showed decreased expression. Several other important genes in this functional category group, such as the polymeric immunoglobulin receptor (Pigr), interferon regulatory factors, immunoglobulinrelated genes immunoglobulin heavy chain complex (Igh), Igh-2 and Igh-6, oligoadenylate synthetases, T-cell specific GTPases and immunity-related GTPase family, M (Irgm), were also found to display decreased expression. The polymeric immunoglobulin receptor and/or the secretory immunoglobulins have been reported to be important in the maintenance of epithelial integrity and mucosal homeostasis in dextran sulfate sodium (DSS)induced mouse model of colitis [72].

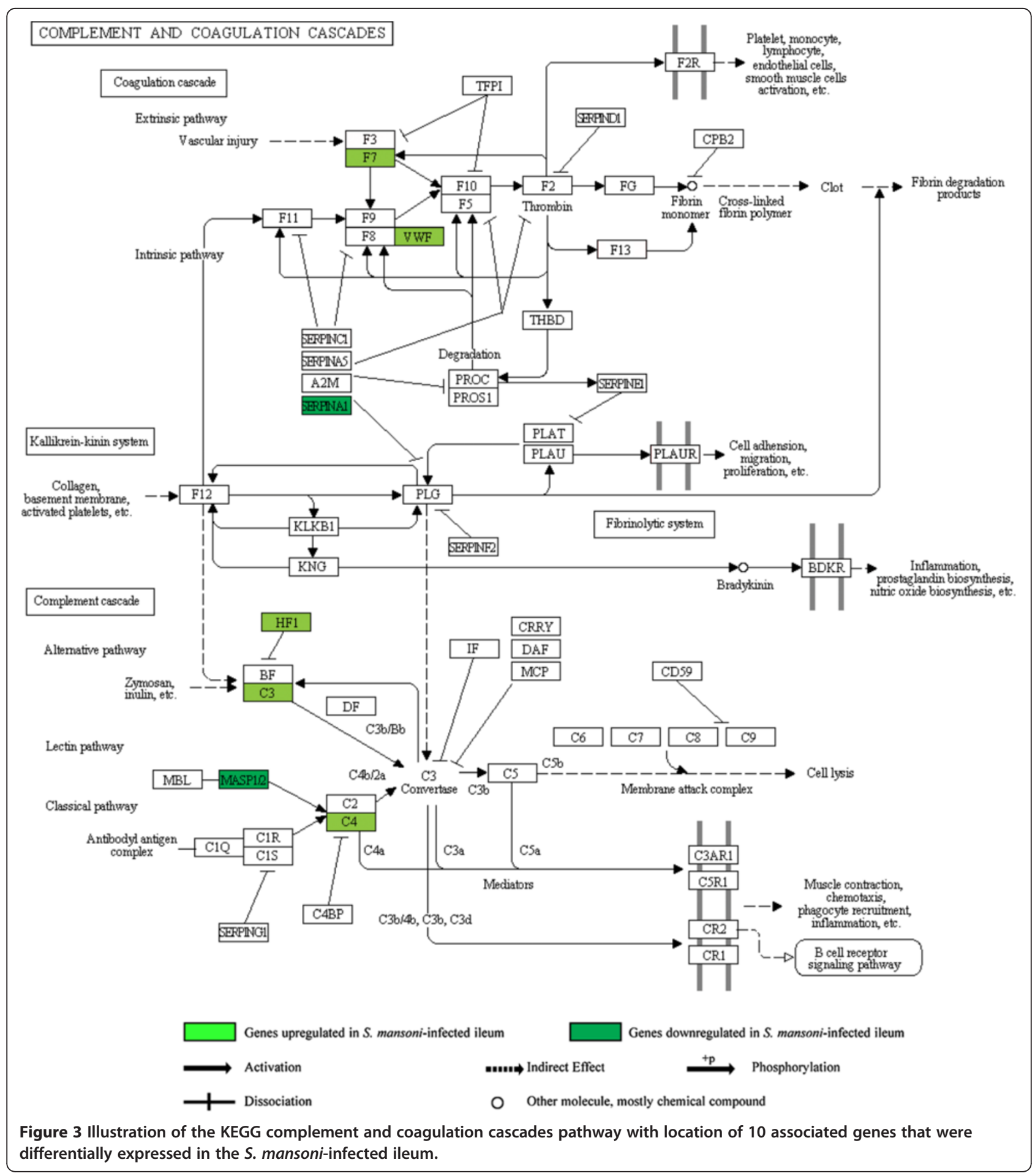




\section{Complement and coagulation cascades pathway and protein activation cascade category group}

(Additional file 1, Additional file 7: Table S1) (Figure 3).

In the $S$. mansoni-infected ileum, changes in this category group suggest complement activation, enhanced innate immune responses, coagulation activation, altered vascular homeostasis and affected acute phase responses, as also observed in some other animal models of intestinal inflammation $[73,74]$. The category group shared 4 genes $(C 3, C 4 b, C f h, F 7)$, all of which showed increased expression. The changes in complement component factor h $(C f h)$ and complement components 3 and $4(C 3$, $C 4 b$ ), indicate enhanced innate immune responses [75,76]. C3 has also been proposed to have roles in directly triggering the degranulation of mast cells, increasing vascular permeability and in smooth muscle contraction [77]. Perhaps related to this complement cascade is our observation of the highly increased

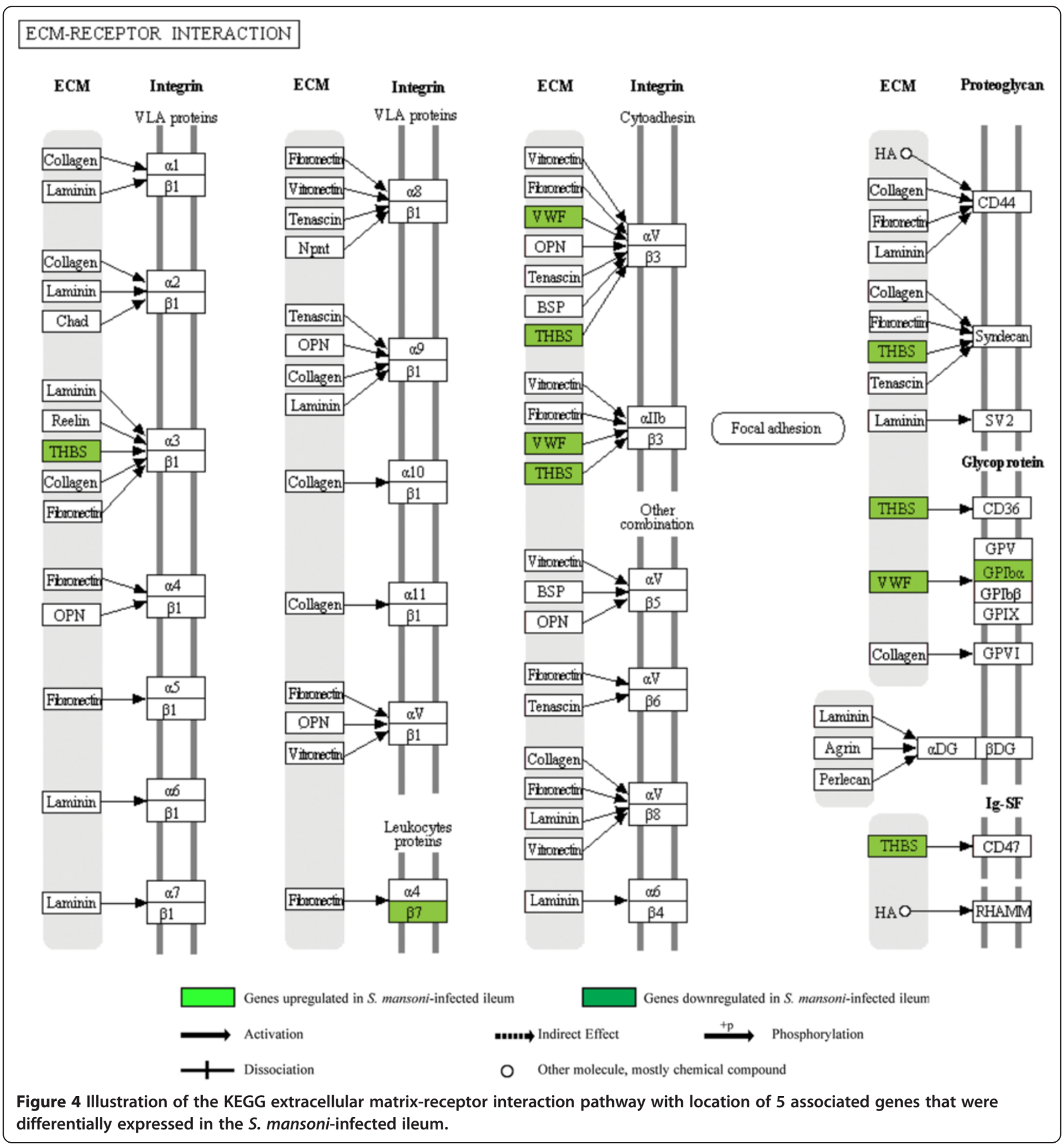


expression of long pentraxin (Ptx3), which is known to be produced by innate immune cells and involved in tuning complement activation and inflammation [78]. Furthermore, the increased expression of coagulation genes Von Willebrand factor homolog $(V w f)$ and coagulation factor VII (F7) implies enhanced coagulation, whereas the decreased expression of the serine peptidase inhibitor genes and regenerating islet-derived 3 alpha (Reg3a) suggests affected acute phase responses, since these genes fall under the acute phase response GO category.

In the TNBS-treated ileum, this category group did not show significant changes.

\section{ECM-receptor interaction pathway and cell adhesion category group}

(Additional file 1, Additional file 7: Table S1) (Figure 4).

In the $S$. mansoni-infected ileum, our results pertaining to this category group suggest altered vascular homeostasis, recruitment of several types of immune cells, enhanced ECM turnover leading to altered adhesion, differentiation, apoptosis, and proliferation, enhanced tissue repair and defects in the epithelial barrier. The ECMreceptor interaction pathway-cell adhesion category group is upregulated in this condition and shared 5 genes (Gp1ba, Itgb7, Thbs3, Thbs4, Vwf), all of which showed increased expression. Increased expression of $V w f$ and the VWF-receptor gene glycoprotein $1 \mathrm{~b}$, alpha polypeptide $(G p 1 b a)$ is mainly suggestive of destabilization of the endothelial barrier, increased vascular permeability and promotion of the inflammatory response [68,74]. The increased expression of integrins Itgae/Cd103 and Itgb7 $(\alpha E \beta 7)$ suggests intraepithelial retention of immune cells in the S. mansoni-infected ileum [79]. As far as other genes in this category group are concerned, the increased expression of integrins Itgae//Cd103 and Itgax (Cd11c) in this model indicates enhanced DC activity $[80,81]$. However, another study reported that CD103, despite displaying an upregulation, is dispensable for intestinal immunity during helminth infection [82]. Increased expression of Siglec 5 points to the accumulation and survival of eosinophils [83] and increased expression of collagenrelated gene Col18a1 points to preservation of integrity of ECM in the basement membrane and hence in wound healing processes in the S. mansoni-infected ileum [84]. Interestingly, two tight junction protein genes claudin 2 and $8(C l d n 2, C l d n 8)$, which are related to cell adhesion, showed decreased expression in this model, indicating alterations in the epithelial paracellular transport [85], which could be associated with the previously reported impaired epithelial barrier function and altered permeability during $S$. mansoni infection [86].

In the TNBS-treated ileum, this category group did not show significant changes.

\section{FceRI signaling pathway and regulation of acute} inflammatory response category group

(Additional file 1, Additional file 7: Table S1).

In the $S$. mansoni-infected ileum, FceRI signaling pathway-regulation of acute inflammatory response category group is upregulated, suggesting enhanced stimulation and amplification of mast cell responses and acute inflammatory responses, such as the Th2 responses, conferring resistance to schistosome eggs. This particular category group shared genes, such as Fcerla and Il4, and these genes showed increased expression. Fc receptor IgE high affinity I $\alpha$ polypeptide $($ Fcer $1 a)$, is known to encode the high-affinity IgE receptor subunit $\alpha$, and to be expressed on mast cells and granulocytes [87]. Acute schistosomiasis is characterized by strong host immune responses with elevated IgE levels correlating with the magnitude of the infection [88]. The interaction of antigen with IgE bound to FceRI triggers mast cells to degranulate, resulting in the release of increased levels of mediators that modulate immune responses and increase smooth muscle contraction and vascular permeability [89]. In line with these findings, we observed increased expression of several mast cellassociated genes, such as the mast cell chymases 1, 2 (Cma1, Cma2), and the mast cell proteases Mcpt1, Mcpt2, Mcpt4 and Mcpt9 in this model, along with the afore-mentioned Fcerla. These findings corroborate the results of another study that reported the predominant recruitment of mucosal mast cells expressing mouse mast cell protease 1 (mMCP-1), a protein encoded by Mcpt9, in acute intestinal schistosomiasis [90]. A crucial role for chymase immunopositive-mast cells has also been reported in the pathophysiology of $C D$ [91]. A positive feedback mechanism between FceRI signaling and IL4 production has been described, and indeed, as discussed earlier, Il4 was increasingly expressed in the S. mansoniinfected ileum [92].

The TNBS-treated ileum did not reveal any significant changes related to this category group, which is not surprising given the known role of the Fce receptor in allergic reactions and defense against parasitic infections, which is not the case in acute TNBS-induced ileitis.

\section{Antigen processing and presentation pathway and antigen processing and presentation (of endogenous antigen, of exogenous peptide antigen and of peptide or polysaccharide antigen via MHC class II) category group} (Additional file 2, Additional file 8: Table S2) (Figure 5).

In the $S$. mansoni-infected ileum, no significant changes were observed in this category group.

In the TNBS-treated ileum, the altered gene expression observed in this category group indicates that antigen processing and presentation are defective in acute TNBS-induced ileitis. This category group shared 13 


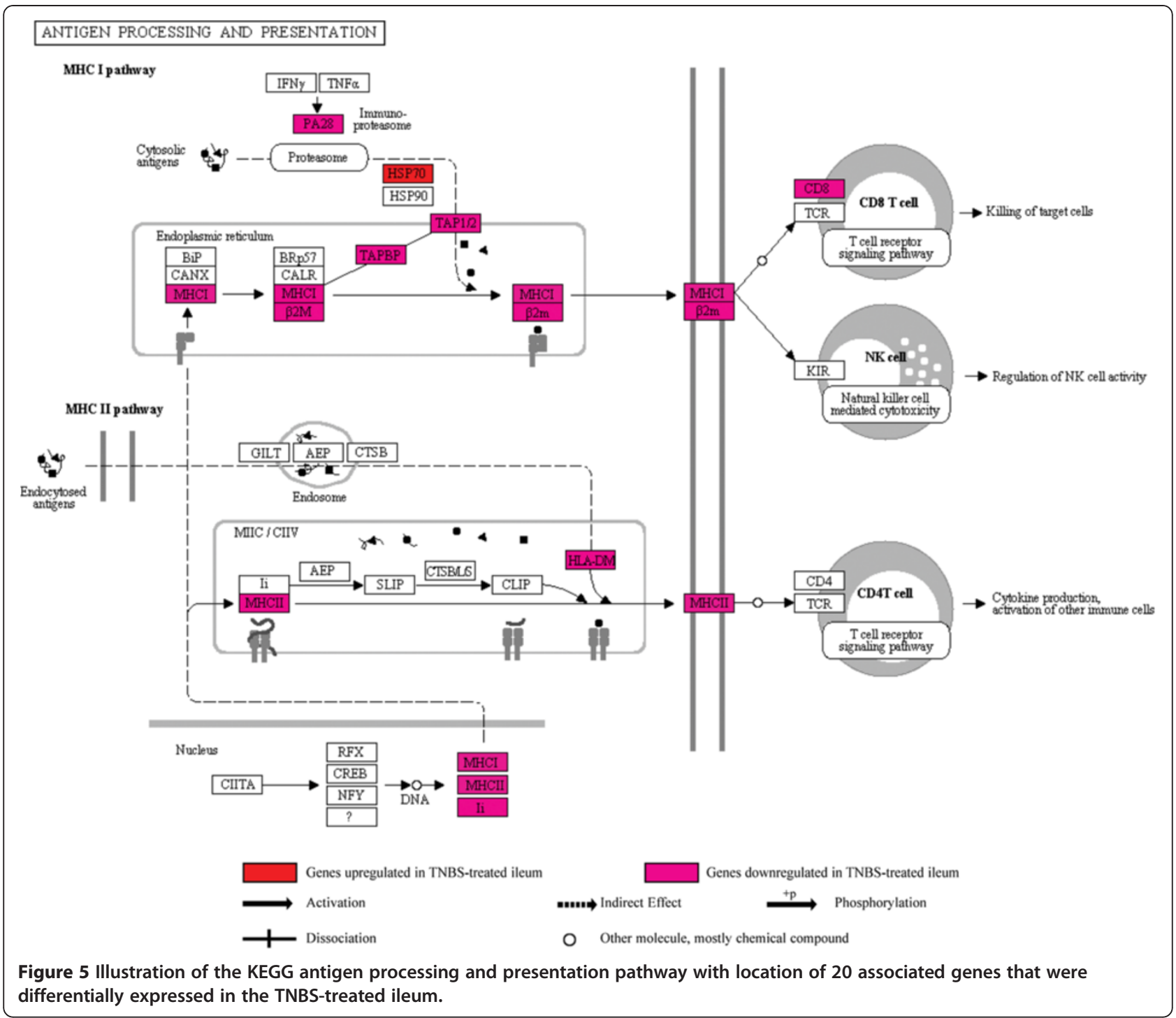

genes (H2-Aa, H2-Ab1, H2-D1, H2-DMa, H2-DMb1, $H 2-D M b 2, H 2-E b 1, H 2-K 1, H 2-O b, H 2-Q 7, H 2-T 23$, Tap2, Tapbp), all of which showed decreased expression. Apart from the above-mentioned MHC class I and class II antigen genes, other genes in this category group, such as $C d 8 a \mathrm{~T}$ cell gene and the $\mathrm{T}$ cell receptor genes Tcra and Tcrg-V4 also showed decreased expression. The earlier reported downregulation of histocompatibility 2, class II antigen $\mathrm{E} \beta(H 2-E b 1)$ and of T cell receptor $\gamma$ in a C57BL/6 mouse model of TNBS-induced colitis is therefore in agreement with our observations [93]. These changes point to defective antigen presentation, decreased cytotoxic $\mathrm{T}$ cell function and the suppression of unconventional regulatory $\mathrm{T}$ cells, such as the resident $\mathrm{TCR} \gamma \delta$ or $\mathrm{TCR} \alpha \beta \mathrm{CD} 8+$ intraepithelial lymphocytes [94]. The decreased expression of heat shock protein 2 (Hspa2), which encodes HSP70A2, along with the increased expression of Hspb1 (HSP25), is indicative of a change in the inherent ability of epithelial cells to defend themselves against cellular stress and injury in acute TNBS-induced ileitis. Hspa2 downregulation also hints at decreased antigen presentation to CD4+ $\mathrm{T}$ cells by antigen presenting cells [95].

\section{Cell adhesion molecules pathway and cell adhesion category group}

(Additional file 2, Additional file 8: Table S2).

In the $S$. mansoni-infected ileum, no significant changes were observed in this category group.

In the TNBS-treated ileum, changes in this category group suggest affected integrity and function of the intestinal epithelial barrier and altered recruitment of several types of immune cells. This particular category group shared 8 genes (Cd6, Cldn2, Cldn8, Itga8, Itgb7, Negr1, Neo1, Ptprf), all of which showed decreased expression. Changes related to these claudin genes have 
Figure 6 Illustration of the KEGG ABC transporters pathway with location of 14 associated genes that were differentially expressed in the TNBS-treated ileum.

also been described in human IBD and in ileitis models $[85,96]$. Decreased expression of integrin beta 7 (Itgb7) together with the afore-mentioned $\mathrm{Ccl} 5$ in this model indicates suppression of lymphocyte recruitment to the intestine [97]. Furthermore, changes in some other genes in this category group, such as the decreased expression of Itgae/Cd103, together with increased expression of Itgam, and of the afore-mentioned Ccl9, indicate the loss of CD103+ intestinal DCs, as seen in murine colitis models [98], and an increased efflux of activated macrophages and DCs to the lymphatics. These changes are perhaps instrumental in the restoration of homeostatic balance through $\mathrm{T}$ cell induction in the TNBS-treated ileum $[59,99]$. Increased expression of genes encoding proteins such as laminin and decreased expression of genes encoding collagens, integrins $\alpha 11$, and tenascin in the cell adhesion category are mainly suggestive of compositional changes in the epithelial basement membrane and interstitial matrix and suppressed wound healing [100,101].

\section{TLR signaling pathway and immune, inflammatory} response category group

(Additional file 2, Additional file 8: Table S2).

In the $S$. mansoni-infected ileum, this TLR-associated category group did not show differential expression, which is in accordance with previous reports stating that antigen secretions of schistosoma eggs, such as the soluble egg antigen, do not require the expression of TLR2 or TLR4 on DCs to induce a polarized Th2 response [102]. Instead, pattern recognition receptor genes other than TLRs, such as the C-type lectin family member genes showed increased expression, confirming that Ctype lectins are likely involved in innate signaling in acute intestinal schistosomiasis [102].

In the TNBS-treated ileum, changes in this category group suggest altered innate immune responses, suppressed barrier protection and aggravated inflammatory responses. The category group shared 6 genes that showed increased expression (Ccl3, Ccl4, Cd14, Il1b, Il6, Tlr6) and 5 shared genes that showed decreased expression (Ccl5, Cxcl9, Irf7, Tlr1, Tlr3). Upregulation of TLR6 has been suggested to lead to pathogen recognition and rapid activation of innate immunity by the induction of inflammatory molecules [103]. The increased expression

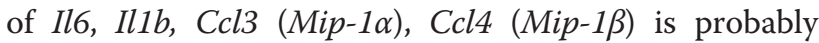
related to this finding. TLR1 expression has been shown to be downregulated in human inflamed colonic epithelial and lamina propria cells and has been linked to responses to TLR2 ligands [104]. A protective role for 
TLR3 signaling has been put forward in the pathogenesis of intestinal inflammation in a murine acute colitis model [105]. Therefore, the observed decreased expression of Tlr3 in our study probably indicates suppression of protective TLR3 signaling. Another interesting gene in this functional category group, which was found to be highly expressed in the TNBS-treated ileum, is secreted phosphoprotein 1 (Spp1), and the protein encoded by this gene is known to be a key immune modulator, stimulating stress responses as well as both pro- and anti-inflammatory processes [106].

\section{$A B C$ transporters pathway and response to chemical stimulus category group}

(Additional file 2, Additional file 8: Table S2) (Figure 6).

In the $S$. mansoni-infected ileum, this category group did not show significant changes.

In the TNBS-treated ileum, changes in this category group suggest a defective efflux of pathogens, drugs and toxins, thereby an impaired epithelial barrier function, an enhanced response to chemical stimuli and altered vascular homeostasis. This category group shared 3 genes (Abca1, Abcb11, Abcc1) with increased expression. Other genes in this functional category group that showed decreased expression included Abca1, Abcb10, $A b c b 11, A b c c 1, A b c c 3, A b c c 4, A b c g 2$, Tap1 and Tap2. The ATP-binding cassette sub-family B member 1, (Abcb1a), which has been observed to change expression in models of colitis and CD [4,107], was not found to be differentially expressed. Other changes related to this category group included those of the coagulation cascade, which seems to be activated given the increased expression of coagulation factors III and XIII $(F 3, F 13)$, the plasminogen activator, the urokinase receptor (Plaur) and some serine (or cysteine) peptidase inhibitor members. These changes lend further link to previous studies that have demonstrated that TNBS administration causes bleeding within the ileal wall [30] and to the existing role of coagulation in the perpetuation of inflammation, as also reported in human and experimental IBD $[68,73]$.

\section{Comparison of the differentially expressed genes in our study with IBD}

Comparison of the differentially expressed genes in the S. mansoni-infected ileum with 32 genes that have previously been shown to exhibit an altered expression pattern in murine models of IBD [4] yielded only 1 overlapping gene (1 upregulated) in the $S$. mansoni-infected ileum; i.e., Timp1. This change in Timp1 expression might be related to an altered epithelial barrier and tissue remodeling [108]. It can be inferred from the comparison that the $S$. mansoni-infected ileum, which is a model of intestinal inflammation not related to IBD, hardly shows any similarity with IBD-associated gene expression. The change in Timp1 expression was also observed in the TNBStreated ileum (see below), implying that activity of this gene is probably not specific to the inflammation type.

In the TNBS-treated ileum, however, comparison with the above-mentioned 32 genes [4], yielded 14 overlapping genes (12 upregulated and 2 downregulated). The higher expressed genes were Il6, Ccl2, Ccl3, Ccl4, Cxcl5, Mmp3, Mmp7, Mmp9, Timp1, S100a8, S100a9 and Ptgs2 and the lower expressed genes were $\mathrm{Ccl} 5$ and $\mathrm{Cxcr3}$. Increased expression of Il6, chemokines and neutrophilassociated genes, are indicative of pro-inflammatory responses and the recruitment of macrophages and granulocytes, as mentioned above. Changes in the expression of matrix metalloproteinases and of the tissue inhibitor of metalloproteinase 1 (Timp1) are probably related to tissue injury, tissue remodeling, angiogenesis, and promotion of leukocyte extravasation observed in this model [108]. Increased expression of Ptgs 2 points to prostaglandin synthesis, as suggested in colonic epithelial cells in IBD and in experimental colitis [4]. It can be inferred from the comparison that although our TNBSinduced ileitis model is an acute insult, 14 genes (out of the 32 genes previously known to show an altered expression pattern in murine models of IBD [4]) showed changed expression; and as such, the expression profile in this model bears considerable relevance to that of IBD. A study of murine TNBS-induced inflammation in colon, conducted by another group, however, revealed very few differentially expressed genes and did not show significant similarities with the expression profile in human IBD [4]. The divergent results in this respect between the latter and our study may originate from several factors, such as the use of a different mouse strain, the application of different dosages, the region studied (colon vs. ileum,) and the type of inflammation (only delayed type hypersensitivity reaction after administration of a second dose vs. acute inflammation in our study). Our study, compared to previously published studies such as the one mentioned above [4], as well as some others [109], highlight the significant differences of results in gene expression in the same inflammatory condition, i.e., in TNBS-induced inflammation, but in other regions of the GI tract and therefore strengthen the need of studies such as ours, to account for the region-specific differences. Furthermore, comparison of the differentially expressed genes in our study with the list of 77 candidate genes located within IBD susceptibility loci [109-112] did not yield any overlapping genes in the S. mansoni-infected ileum, whereas 9 genes (4 up and 5 down) were overlapping in the TNBS-treated ileum (Table 1).

This brief comparative analysis with IBD-related or IBD-associated genes, clearly demonstrates that gene 
Table 1 Comparison of the differentially expressed genes in the S. mansoni-infected and the TNBS-treated ileum with candidate genes located within IBD susceptibility loci

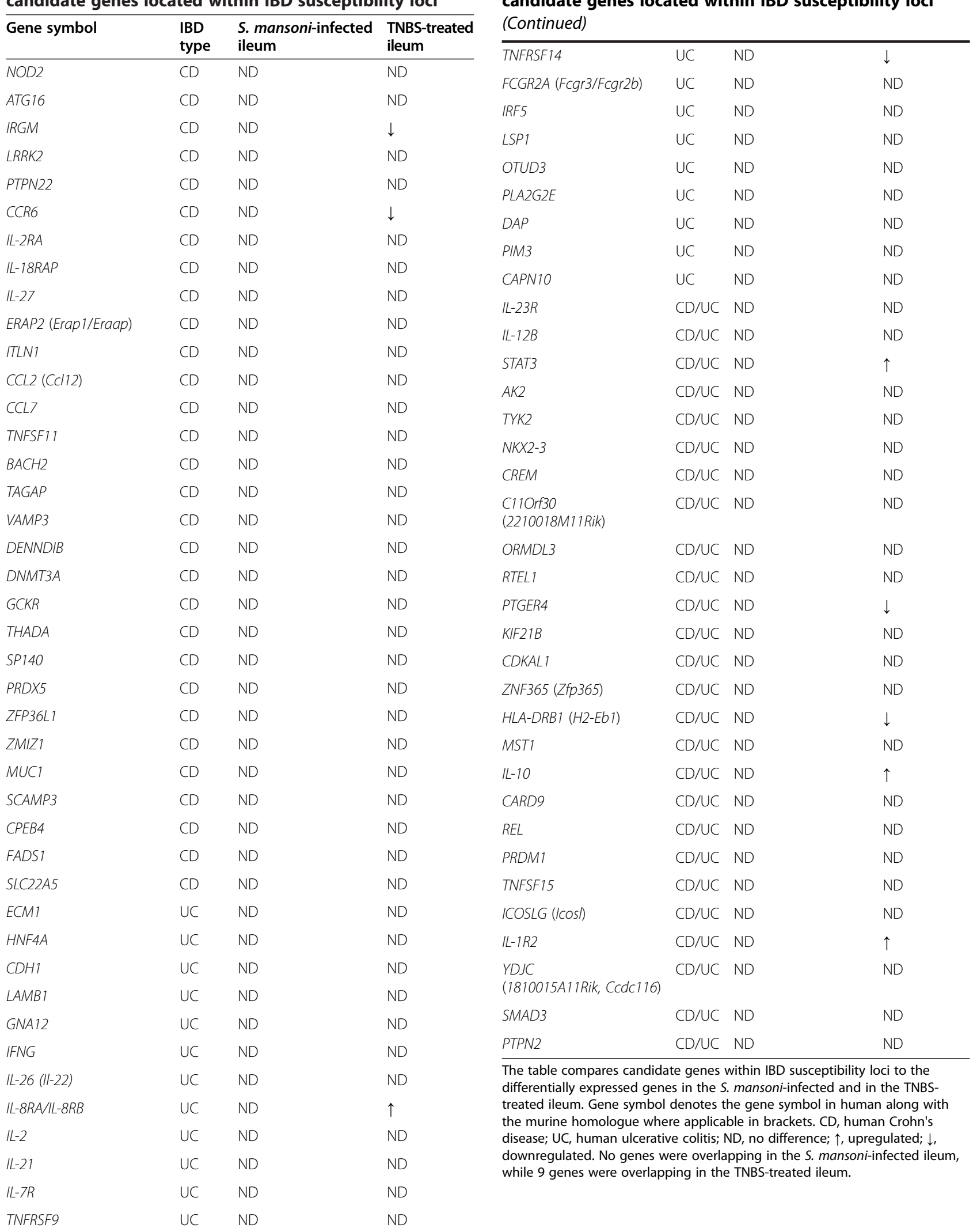

Table 1 Comparison of the differentially expressed genes in the S. mansoni-infected and the TNBS-treated ileum with candidate genes located within IBD susceptibility loci 
expression aspects of the TNBS-induced ileitis model in our study have noticeable relevance to IBD.

\section{Conclusions}

The present study in mouse is the first to identify changes in whole-genome gene expression in the acutely inflamed ileum of two murine models of intestinal inflammation and to characterize the biological relevance related to these changes. The results demonstrate that gene expression profiles and patterns are considerably distinct in the two models owing to the differences in etiology. The results suggest that acute intestinal schistosomiasis shows enhanced Th2 responses, whereas acute TNBS-induced ileitis exhibits Th17 cell priming. Although two functional category groups (cytokine-cytokine receptor interaction pathway-immune response category group and intestinal immune network for IgA production pathway-immune response category group) are common to both models of inflammation, the predisposing genes are largely unique within the pathways. Other functional category groups, such as the complement and coagulation cascades pathway-protein activation cascade, ECM-receptor interaction pathway-cell adhesion, Fc epsilon RI signaling pathway-acute inflammatory response are unique to the acute intestinal schistosomiasis model, and are upregulated, whereas antigen processing and presentation, cell adhesion, TLR signaling pathway-immune and inflammatory response and $\mathrm{ABC}$ transporters pathway-response to chemical stimulus category groups are unique to the acute TNBS-induced ileitis model, and are more bent towards downregulation. All the above findings underscore the distinct gene expression patterns in the two inflammation models of our study. Acute intestinal schistosomiasis seems to promote a number of specific processes, such as complement-mediated innate immunity, protein activation, ECM turnover, acute inflammatory responses as well as antibody-mediated defense responses. Acute TNBSinduced ileitis seems to be associated with aspecific proinflammatory responses, alterations in TLR-mediated innate immunity, defective antigen processing and presentation, angiogenesis and anti-angiogenesis, reduced cytotoxic $\mathrm{T}$ cell function, compositional changes in the epithelial basement membrane and interstitial matrix, enhanced tissue remodeling and a defective antibody line of defense. Altered vascular homeostasis and impaired epithelial barrier function seem to be common features in both models of inflammation. The gene expression profiles in acute TNBS-induced ileitis show considerable relevance to those of IBD. This is of course no complete resemblance, but insight into the differences between animal models and the human situation is equally important. Furthermore, better characterization of animal models leads to better interpretation of results concerning translational research. In addition to the above findings, the present study revealed a differentially expressed gene list that includes many genes for which pathways or functional annotations have not yet been defined. Therefore, the comprehensive differential gene list together with the functional grouping, in particular, provided by this study offers a valuable resource and a starting point to explore extended functional networks, new targets or a specific novel pathway in greater detail related to small bowel inflammation. Future application of these analyses to other ileal inflammation models, including chronic stages and other time points, will yield greater insights and lead to a better understanding of ileal inflammation.

\section{Additional files}

\begin{abstract}
Additional file 1: Complete list of differentially expressed genes in the $S$. mansoni-infected ileum. Differentially expressed genes in the $S$. mansoni-infected ileum are represented with their ID (Agilent probe ID), GB_ACC (gene bank accession), GENE_SYMBOL (gene symbol), GENE_NAME (gene name), $\log _{2} F C$ ( $\log _{2}$ fold change), $p$-value and adjusted $p$-value. Upregulated genes are shown in red; downregulated genes are shown in green; overlapping genes that changed in the same direction as in the TNBS-treated ileum are displayed in bold; overlapping genes that changed in the opposite direction to that of the TNBS-treated ileum are underlined. All genes listed in this file showed significant differential expression ( $p$-value $<0.05$, adjusted $p$-value $<0.1$ and $\log _{2}$ fold change $>1$ or $<-1$ ) relative to controls.
\end{abstract}

Additional file 2: Complete list of differentially expressed genes in the TNBS-treated ileum. Differentially expressed genes in the TNBStreated ileum are represented with their ID (Agilent probe ID), GB_ACC (gene bank accession), GENE_SYMBOL (gene symbol), GENE_NAME (gene name), $\log _{2}$ FC ( $\log _{2}$ fold change), p-value and adjusted p-value. Upregulated genes are shown in red; downregulated genes are shown in green; overlapping genes that changed in the same direction as in the $S$. mansoni-infected ileum are displayed in bold; overlapping genes that changed in the opposite direction to that of the S. mansoni-infected ileum are underlined. All genes listed in this file showed significant differential expression ( $p$-value $<0.05$, adjusted $p$-value $<0.1$ and $\log _{2}$ fold change $>1$ or $<-1$ ) relative to controls.

Additional file 3: Complete list of differentially expressed genes that could be annotated with a KEGG pathway term and/or a GO term in the S. mansoni-infected ileum. Differentially expressed genes are listed together with their KEGG and/or GO annotations. GenID (Ensembl ID), Symbol (gene symbol), \#GO-Terms Associated (number of GO terms associated), Percent GO-Terms Associated, UID-str (unique Agilent ID), GO Names (names of associated terms). Of the 207 unique genes that were differentially expressed in the S. mansoni-infected ileum, 42 genes could be linked to a KEGG pathway term and 144 genes could be linked to a GO term.

Additional file 4: Complete list of differentially expressed genes that could be annotated with a KEGG pathway term and/or a GO term in the TNBS-treated ileum. Differentially expressed genes are listed together with their KEGG and/or GO annotations. GenID (Ensembl ID), Symbol (gene symbol), \#GO-Terms Associated (number of GO terms associated), Percent GO-Terms Associated, UID-str (unique Agilent ID), GO Names (names of associated terms). Of the 1417 unique genes that were differentially expressed in the TNBS-treated ileum, 232 genes could be linked to a KEGG pathway term and 1050 genes could be linked to a GO term.

Additional file 5: KEGG pathways and GO (biological process) categories that were over-represented in the $S$. mansoni-infected ileum. KEGG pathways and GO (biological process) categories that were over-represented in the S. mansoni-infected ileum are represented with their MAPP Name (KEGG pathway name), Z score (z-score), AdjustedP 
(adjusted permutation p-value calculated using the Benjamini-Hochberg method)), gene symbols, AVG-logFC (average $\log _{2} F C$ for each enriched term calculated based on associated differential genes) and AVG-adj.P. Val (average adjusted $\mathrm{p}$-value for each enriched term calculated based on associated differential genes, GOID (Gene ontology ID), GO Name (name of GO category). Threshold criteria for over-representation: z-score > 2, at least three genes from the input list in the enriched category, an adjusted permutation $\mathrm{p}$-value $\leq 0.35$. Top-scoring functional category groups for KEGG pathway and GO categories are shown in colour as follows: Cytokine-cytokine receptor interaction pathway and immune, defense response category group. Intestinal immune network for IgA production pathway and immune response category group.

Complement and coagulation cascades pathway and protein activation cascade category group. ECM-receptor interaction pathway and cell adhesion category group. Fc epsilon RI signaling pathway and acute inflammatory response category. (Note: Although immune response category is common to more than one category group, it is coloured only once).

Additional file 6: KEGG pathways and GO (biological process) categories that were over-represented in the TNBS-treated ileum. KEGG pathways and GO (biological process) categories that were overrepresented in the TNBS-treated ileum are represented with their MAPP Name (KEGG pathway name), Z score (z-score), AdjustedP (adjusted permutation $p$-value calculated using the Benjamini-Hochberg method)), gene symbols, AVG-logFC (average $\log _{2} F C$ for each enriched term calculated based on associated differential genes) and AVG-adj.P. Val (average adjusted p-value for each enriched term calculated based on associated differential genes, GOID (Gene ontology ID), GO Name (name of GO category). Threshold criteria for over-representation: $z$-score $>2$, at least three genes from the input list in the enriched category, an adjusted permutation $p$-value $\leq 0.35$. Top-scoring functional category groups for KEGG pathway and GO categories are shown in colour as follows: Cytokine-cytokine receptor interaction pathway and inflammatory, immune response category group. Intestinal immune network for IgA production pathway and immune response category group. Antigen processing and presentation pathway and antigen processing and presentation category group. Cell adhesion molecules pathway and cell adhesion category group. Toll-like receptor signaling pathway and immune and inflammatory response category group. $A B C$ transporters pathway and response to chemical stimulus category group. (Note: Although immune, inflammatory response categories are common to more than one category group, they are coloured only once).

Additional file 7: Table S1. High scoring functional category groups in the S. mansoni-infected ileum.

Additional file 8: Table S2. High scoring functional category groups in the TNBS-treated ileum.

\section{Abbreviations}

CD: human Crohn's disease; DCs: dendritic cells; ECM: extracellular matrix; FcERI: Fc epsilon receptor I; Gl: gastrointestinal; GO: gene ontology; IBD: inflammatory bowel diseases; IgA: immunoglobulin A; KEGG: Kyoto Encyclopedia for Genes and Genomes; $\log _{2} \mathrm{FC}: \log _{2}$ fold change; MHC: major histocompatibility complex; TLR: Toll-like receptor; TNBS: 2,4,6-trinitrobenzene sulfonate; UC: human ulcerative colitis..

\section{Competing interests}

The authors declare that they have no competing interests.

\section{Authors' contributions}

LRA, DK, RB and LV conceived the study, designed the experiments, prepared the samples and performed the experiments. LRA, DK and RB conducted the data analysis and wrote the initial manuscript. LRA, LV, DA, LVN and JPT contributed to the bioinformatics analysis and manuscript editing. LVN and JPT provided critical advice and conceptual guidance for the study. All authors read and approved the final manuscript.

\section{Acknowledgements}

The authors greatly appreciate the assistance of Pascal Torfs and Dominique De Rijck, and thank the entire staff of the Laboratory of Cell biology and
Histology as well as of the Laboratory of Ecophysiology, Biochemistry \& Toxicology for their excellent technical assistance. We would also like to acknowledge the assistance of Danny Vindevogel in editing the text. This study was funded by a grant of the Flemish Organization for Scientific Research (FWO G.0179.08) and by a TOP-BOF (2008-2011) project of the University of Antwerp.

\section{Author details}

'Department of Veterinary Sciences, Laboratory of Cell Biology and Histology, University of Antwerp, Groenenborgerlaan 171, Antwerp B-2020, Belgium. ${ }^{2}$ Department of Biology, Laboratory of Ecophysiology, Biochemistry and Toxicology, University of Antwerp, Groenenborgerlaan 171, Antwerp B-2020, Belgium. ${ }^{3}$ Faculty of Medicine and Health Sciences, Laboratory of Human Anatomy and Embryology, University of Antwerp, Groenenborgerlaan 171, Antwerp B-2020, Belgium. ${ }^{4}$ Department of Veterinary Sciences, Laboratory of Physiology and Biochemistry of Domestic Animals, University of Antwerp, Universiteitsplein 1, Antwerp B-2610, Belgium.

Received: 13 January 2012 Accepted: 18 July 2012

Published: 6 August 2012

\section{References}

1. Costello CM, Mah N, Hasler R, Rosenstiel P, Waetzig GH, Hahn A, Lu T, Gurbuz Y, Nikolaus S, Albrecht M, Hampe J, Lucius R, Kloppel G, Eickhoff H, Lehrach H, Lengauer T, Schreiber S: Dissection of the inflammatory bowel disease transcriptome using genome-wide CDNA microarrays. PLOS Med 2005, 2:e199.

2. Dieckgraefe BK, Stenson WF, Korzenik JR, Swanson PE, Harrington CA: Analysis of mucosal gene expression in inflammatory bowel disease by parallel oligonucleotide arrays. Physiol Genomics 2000, 4:1-11.

3. Lawrance IC, Fiocchi C, Chakravarti S: Ulcerative colitis and Crohn's disease: distinctive gene expression profiles and novel susceptibility candidate genes. Hum Mol Genet 2001, 10:445-456.

4. te Velde AA, de KF, Sterrenburg E, Pronk I, ten Kate FJ, Hommes DW, van Deventer SJ: Comparative analysis of colonic gene expression of three experimental colitis models mimicking inflammatory bowel disease. Inflamm Bowel Dis 2007, 13:325-330.

5. Wu F, Dassopoulos T, Cope L, Maitra A, Brant SR, Harris ML, Bayless TM, Parmigiani G, Chakravarti S: Genome-wide gene expression differences in Crohn's disease and ulcerative colitis from endoscopic pinch biopsies: insights into distinctive pathogenesis. Inflamm Bowel Dis 2007, 13:807-821.

6. Noble CL, Abbas AR, Lees CW, Cornelius J, Toy K, Modrusan Z, Clark HF, Arnott ID, Penman ID, Satsangi J, Diehl L: Characterization of intestinal gene expression profiles in Crohn's disease by genome-wide microarray analysis. Inflamm Bowel Dis 2010, 16:1717-1728.

7. Caprilli R: Why does Crohn's disease usually occur in terminal ileum? J Crohns Colitis 2008, 2:352-356.

8. Haskell H, Andrews CW Jr, Reddy SI, Dendrinos K, Farraye FA, Stucchi AF, Becker JM, Odze RD: Pathologic features and clinical significance of "backwash" ileitis in ulcerative colitis. Am J Surg Pathol 2005, 29:1472-1481.

9. Yamamoto T, Maruyama Y, Umegae S, Matsumoto K, Saniabadi AR: Mucosal inflammation in the terminal ileum of ulcerative colitis patients: endoscopic findings and cytokine profiles. Dig Liver Dis 2008, 40:253-259.

10. Barnes SL, Vidrich A, Wang ML, Wu GD, Cominelli F, Rivera-Nieves J, Bamias G, Cohn SM: Resistin-like molecule beta (RELMbeta/FIZZ2) is highly expressed in the ileum of SAMP1/YitFc mice and is associated with initiation of ileitis. J Immunol 2007, 179:7012-7020.

11. McNamee EN, Wermers JD, Masterson JC, Collins CB, Lebsack MD, Fillon S, Robinson ZD, Grenawalt J, Lee JJ, Jedlicka P, Furuta GT, Rivera-Nieves J: Novel model of $\mathrm{T}(\mathrm{H}) 2$-polarized chronic ileitis: The SAMP1 mouse. Inflamm Bowel Dis 2009.

12. Pizarro TT, Arseneau KO, Bamias G, Cominelli F: Mouse models for the study of Crohn's disease. Trends Mol Med 2003, 9:218-222.

13. Pizarro TT, Pastorelli L, Bamias G, Garg RR, Reuter BK, Mercado JR, Chieppa M, Arseneau KO, Ley K, Cominelli F: SAMP1/YitFc mouse strain: A spontaneous model of Crohn's disease-like ileitis. Inflamm Bowel Dis 2011.

14. Bates MD, Erwin CR, Sanford LP, Wiginton D, Bezerra JA, Schatzman LC, Jegga AG, Ley-Ebert C, Williams SS, Steinbrecher KA, Warner BW, Cohen MB, Aronow BJ: Novel genes and functional relationships in the adult mouse 
gastrointestinal tract identified by microarray analysis. Gastroenterology 2002, 122:1467-1482.

15. Bogaert S, Laukens D, Peeters H, Melis L, Olievier K, Boon N, Verbruggen G, Vandesompele J, Elewaut D, De Vos M: Differential mucosal expression of Th17-related genes between the inflamed colon and ileum of patients with inflammatory bowel disease. BMC Immunol 2010, 11:61.

16. Gryseels B, Polman K, Clerinx J, Kestens L: Human schistosomiasis. Lancet 2006, 368:1106-1118.

17. Bogers J, Moreels T, De Man J, Vrolix G, Jacobs W, Pelckmans P, Van Marck E: Schistosoma mansoni infection causing diffuse enteric inflammation and damage of the enteric nervous system in the mouse small intestine. Neurogastroenterol Motil 2000, 12:431-440.

18. Domingo EO, Warren KS: Pathology and pathophysiology of the small intestine in murine schistosomiasis mansoni, including a review of the literature. Gastroenterology 1969, 56:231-240.

19. Rocha MO, Pedroso ER, Lambertucci JR, Greco DB, Rocha RL, Rezende DF, Neves J: Gastro-intestinal manifestations of the initial phase of schistosomiasis mansoni. Ann Trop Med Parasitol 1995, 89:271-278.

20. Elliott DE, Li J, Blum A, Metwali A, Qadir K, Urban JF Jr, Weinstock JV: Exposure to schistosome eggs protects mice from TNBS-induced colitis. Am J Physiol Gastrointest Liver Physiol 2003, 284:G385-G391.

21. Ruyssers NE, De Winter BY, De Man JG, Loukas A, Pearson MS, Weinstock JV, Van den Bossche RM, Martinet W, Pelckmans PA, Moreels TG: Therapeutic potential of helminth soluble proteins in TNBS-induced colitis in mice. Inflamm Bowel Dis 2009, 15:491-500.

22. Weinstock JV, Elliott D: The somatostatin immunoregulatory circuit present at sites of chronic inflammation. Eur J Endocrinol 2000, 143(Suppl 1):S15-S19.

23. Stavitsky AB: Regulation of granulomatous inflammation in experimental models of schistosomiasis. Infect Immun 2004, 72:1-12.

24. Elson CO, Sartor RB, Tennyson GS, Riddell RH: Experimental models of inflammatory bowel disease. Gastroenterology 1995, 109:1344-1367.

25. Moreels TG, De Man JG, Dick JM, Nieuwendijk RJ, De Winter BY, Lefebvre RA, Herman AG, Pelckmans PA: Effect of TNBS-induced morphological changes on pharmacological contractility of the rat ileum. Eur $J$ Pharmacol 2001, 423:211-222.

26. te Velde AA, Verstege MI, Hommes DW: Critical appraisal of the current practice in murine TNBS-induced colitis. Inflamm Bowel Dis 2006, 12:995-999.

27. Hoshino S, Inaba M, Iwai H, Ito T, Li M, Gershwin ME, Okazaki K, Ikehara S: The role of dendritic cell subsets in 2,4,6-trinitrobenzene sulfonic acidinduced ileitis. J Autoimmun 2010, 34:380-389.

28. Yolles TK, Moore DV, Meleney HE: Post-cercarial development of Schistosoma mansoni in the rabbit and hamster after intraperitoneal and percutaneous infection. J Parasitol 1949, 35:276-294.

29. Avula LR, Buckinx R, Alpaerts K, Costagliola A, Adriaensen D, Van Nassauw L, Timmermans JP: The effect of inflammation on the expression and distribution of the MAS-related gene receptors MrgE and MrgF in the murine ileum. Histochem Cell Biol 2011, 136:569-585.

30. Pontell L, Castelucci P, Bagyanszki M, Jovic T, Thacker M, Nurgali K, Bron R, Furness JB: Structural changes in the epithelium of the small intestine and immune cell infiltration of enteric ganglia following acute mucosal damage and local inflammation. Virchows Arch 2009, 455:55-65.

31. Vergauwen $L$, Benoot D, Blust R, Knapen D: Long-term warm or cold acclimation elicits a specific transcriptional response and affects energy metabolism in zebrafish. Comp Biochem Physiol A Mol Integr Physiol 2010, 157:149-157.

32. Knapen D, Vergauwen L, Laukens K, Blust R: Best practices for hybridization design in two-colour microarray analysis. Trends Biotechnol 2009, 27:406-414.

33. Smyth GK: Linear models and empirical bayes methods for assessing differential expression in microarray experiments. Stat Appl Genet Mol Biol 2004, 3:3.

34. Wettenhall JM, Smyth GK: limmaGUI: a graphical user interface for linear modeling of microarray data. Bioinformatics 2004, 20:3705-3706.

35. De WM, Keil D, Remmerie N, van d V, van den Brandhof EJ, Knapen D, Witters E, De CW: Molecular targets of TBBPA in zebrafish analysed through integration of genomic and proteomic approaches. Chemosphere 2008, 74:96-105.

36. Sclep G, Allemeersch J, Liechti R, De MB, Beynon J, Bhalerao R, Moreau Y Nietfeld W, Renou JP, Reymond P, Kuiper MT, Hilson P: CATMA, a comprehensive genome-scale resource for silencing and transcript profiling of Arabidopsis genes. BMC Bioinformatics 2007, 8:400.
37. Huber W, Von HA, Sultmann H, Poustka A, Vingron M: Variance stabilization applied to microarray data calibration and to the quantification of differential expression. Bioinformatics 2002, 18(Suppl 1):S96-104.

38. Ritchie ME, Silver J, Oshlack A, Holmes M, Diyagama D, Holloway A, Smyth GK: A comparison of background correction methods for two-colour microarrays. Bioinformatics 2007, 23:2700-2707.

39. Hardy J, Singleton A: Genomewide association studies and human disease. N Engl J Med 2009, 360:1759-1768.

40. Curtis RK, Oresic M, Vidal-Puig A: Pathways to the analysis of microarray data. Trends Biotechnol 2005, 23:429-435.

41. Salomonis N, Nelson B, Vranizan K, Pico AR, Hanspers K, Kuchinsky A, Ta L, Mercola M, Conklin BR: Alternative splicing in the differentiation of human embryonic stem cells into cardiac precursors. PLoS Comput Biol 2009, 5:e1000553.

42. Kanehisa M, Goto S: KEGG: kyoto encyclopedia of genes and genomes. Nucleic Acids Res 2000, 28:27-30.

43. Kanehisa M, Goto S, Hattori M, Aoki-Kinoshita KF, Itoh M, Kawashima S, Katayama T, Araki M, Hirakawa M: From genomics to chemical genomics: new developments in KEGG. Nucleic Acids Res 2006, 34:D354-D357.

44. Doniger SW, Salomonis N, Dahlquist KD, Vranizan K, Lawlor SC, Conklin BR: MAPPFinder: using Gene Ontology and GenMAPP to create a global gene-expression profile from microarray data. Genome Biol 2003, 4:R7.

45. Dahlquist KD, Salomonis N, Vranizan K, Lawlor SC, Conklin BR: GenMAPP, a new tool for viewing and analyzing microarray data on biological pathways. Nat Genet 2002, 31:19-20.

46. Boros DL: The role of cytokines in the formation of the schistosome egg granuloma. Immunobiology 1994, 191:441-450.

47. Cheever AW, Lenzi JA, Lenzi HL, Andrade ZA: Experimental models of Schistosoma mansoni infection. Mem Inst Oswaldo Cruz 2002, 97:917-940.

48. Dixon H, Blanchard C, Deschoolmeester ML, Yuill NC, Christie JW, Rothenberg ME, Else KJ: The role of Th2 cytokines, chemokines and parasite products in eosinophil recruitment to the gastrointestinal mucosa during helminth infection. Eur J Immunol 2006, 36:1753-1763.

49. Souza AL, Souza PR, Pereira CA, Fernandes A, Guabiraba R, Russo RC, Vieira $L Q$, Correa A Jr, Teixeira MM, Negrao-Correa D: Experimental infection with Schistosoma mansoni in CCR5-deficient mice is associated with increased disease severity, as CCR5 plays a role in controlling granulomatous inflammation. Infect Immun 2011, 79:1741-1749.

50. Artis D, Wang ML, Keilbaugh SA, He W, Brenes M, Swain GP, Knight PA, Donaldson DD, Lazar MA, Miller HR, Schad GA, Scott P, Wu GD: RELMbeta/ $\mathrm{FIZZ2}$ is a goblet cell-specific immune-effector molecule in the gastrointestinal tract. Proc Natl Acad Sci U S A 2004, 101:13596-13600.

51. Kawada M, Hachiya Y, Arihiro A, Mizoguchi E: Role of mammalian chitinases in inflammatory conditions. Keio J Med 2007, 56:21-27.

52. Chen Z, Laurence A, O'Shea JJ: Signal transduction pathways and transcriptional regulation in the control of Th17 differentiation. Semin Immunol 2007, 19:400-408.

53. Ivanov II, Zhou L, Littman DR: Transcriptional regulation of Th17 cell differentiation. Semin Immunol 2007, 19:409-417.

54. Sanchez-Munoz F, Dominguez-Lopez A, Yamamoto-Furusho JK: Role of cytokines in inflammatory bowel disease. World J Gastroenterol 2008, 14:4280-4288.

55. Abraham C, Cho J: Interleukin-23/Th17 pathways and inflammatory bowel disease. Inflamm Bowel Dis 2009, 15:1090-1100.

56. Lochner M, Peduto L, Cherrier M, Sawa S, Langa F, Varona R, Riethmacher D, Si-Tahar M, Di Santo JP, Eberl G: In vivo equilibrium of proinflammatory $\mathrm{IL}-17+$ and regulatory IL-10+ Foxp3+ RORgamma $\mathrm{t}+\mathrm{T}$ cells. J Exp Med 2008, 205:1381-1393.

57. Wei L, Laurence A, Elias KM, O'Shea JJ: IL-21 is produced by Th17 cells and drives IL-17 production in a STAT3-dependent manner. J Biol Chem 2007, 282:34605-34610.

58. Denning TL, Wang YC, Patel SR, Williams IR, Pulendran B: Lamina propria macrophages and dendritic cells differentially induce regulatory and interleukin 17-producing T cell responses. Nat Immunol 2007, 8:1086-1094.

59. Zhao X, Sato A, Dela Cruz CS, Linehan M, Luegering A, Kucharzik T, Shirakawa AK, Marquez G, Farber JM, Williams I, Iwasaki A: CCL9 is secreted by the follicle-associated epithelium and recruits dome region Peyer's patch CD11b+ dendritic cells. J Immuno/ 2003, 171:2797-2803.

60. Nacken W, Roth J, Sorg C, Kerkhoff C: S100A9/S100A8: Myeloid representatives of the $\mathrm{S} 100$ protein family as prominent players in innate immunity. Microsc Res Tech 2003, 60:569-580. 
61. Abi Abdallah DS, Egan CE, Butcher BA, Denkers EY: Mouse neutrophils are professional antigen-presenting cells programmed to instruct Th1 and Th17 T-cell differentiation. Int Immunol 2011, 23:317-326.

62. Tokumasa N, Suto A, Kagami S, Furuta S, Hirose K, Watanabe N, Saito Y, Shimoda K, Iwamoto I, Nakajima H: Expression of Tyk2 in dendritic cells is required for IL-12, IL-23, and IFN-gamma production and the induction of Th1 cell differentiation. Blood 2007, 110:553-560.

63. Yang $Y, X u$ J, Niu $Y$, Bromberg JS, Ding $Y$ : T-bet and eomesodermin play critical roles in directing $T$ cell differentiation to Th1 versus Th17. J Immunol 2008, 181:8700-8710.

64. Qin S, Rottman JB, Myers $P$, Kassam N, Weinblatt M, Loetscher M, Koch AE, Moser B, Mackay CR: The chemokine receptors CXCR3 and CCR5 mark subsets of T cells associated with certain inflammatory reactions. J Clin Invest 1998, 101:746-754.

65. Wang D, DuBois RN, Richmond A: The role of chemokines in intestinal inflammation and cancer. Curr Opin Pharmacol 2009, 9:688-696.

66. Schmitz J, Owyang A, Oldham E, Song Y, Murphy E, McClanahan TK, Zurawski G, Moshrefi M, Qin J, Li X, Gorman DM, Bazan JF, Kastelein RA: IL-33, an interleukin-1-like cytokine that signals via the IL-1 receptorrelated protein ST2 and induces T helper type 2-associated cytokines. Immunity 2005, 23:479-490.

67. Oboki K, Ohno T, Kajiwara N, Arae K, Morita H, Ishii A, Nambu A, Abe T, Kiyonari H, Matsumoto K, Sudo K, Okumura K, Saito H, Nakae S: IL-33 is a crucial amplifier of innate rather than acquired immunity. Proc Natl Acad Sci U S A 2010, 107:18581-18586.

68. Gutierrez LS: The Role of Thrombospondin 1 on Intestinal Inflammation and Carcinogenesis. Biomark Insights 2008, 2008:171-178.

69. Esplugues E, Huber S, Gagliani N, Hauser AE, Town T, Wan YY, O'Connor W Jr, Rongvaux A, Van RN, Haberman AM, Iwakura Y, Kuchroo VK, Kolls JK, Bluestone JA, Herold KC, Flavell RA: Control of TH17 cells occurs in the small intestine. Nature 2011, 475:514-518.

70. Read S, Malmstrom V, Powrie F: Cytotoxic T lymphocyte-associated antigen 4 plays an essential role in the function of CD25(+)CD4(+) regulatory cells that control intestinal inflammation. J Exp Med 2000 192:295-302.

71. Crabtree JE, Pullar CE, Trejdosiewicz LK, Wilson RA: Murine intestinal humoral responses in chronic Schistosoma mansoni infections. Scand J Immunol 1992, 35:361-367.

72. Murthy AK, Dubose CN, Banas JA, Coalson JJ, Arulanandam BP: Contribution of polymeric immunoglobulin receptor to regulation of intestinal inflammation in dextran sulfate sodium-induced colitis. J Gastroenterol Hepatol 2006, 21:1372-1380.

73. Danese $S$ : Role of the vascular and lymphatic endothelium in the pathogenesis of inflammatory bowel disease: 'brothers in arms'. Gut 2011, 60:998-1008.

74. Petri B, Broermann A, Li H, Khandoga AG, Zarbock A, Krombach F, Goerge T, Schneider SW, Jones C, Nieswandt B, Wild MK, Vestweber D: von Willebrand factor promotes leukocyte extravasation. Blood 2010, 116:4712-4719.

75. Fraczek LA, Martin BK: Transcriptional control of genes for soluble complement cascade regulatory proteins. Mol Immunol 2010, 48:9-13.

76. Duncan RC, Wijeyewickrema LC, Pike RN: The initiating proteases of the complement system: controlling the cleavage. Biochimie 2008, 90:387-395.

77. Peng Q, Li K, Sacks SH, Zhou W: The role of anaphylatoxins C3a and C5a in regulating innate and adaptive immune responses. Inflamm Allergy Drug Targets 2009, 8:236-246.

78. Deban L, Bottazzi B, Garlanda C, de la Torre YM, Mantovani A: Pentraxins: multifunctional proteins at the interface of innate immunity and inflammation. Biofactors 2009, 35:138-145.

79. Cepek KL, Parker CM, Madara JL, Brenner MB: Integrin alpha E beta 7 mediates adhesion of T lymphocytes to epithelial cells. J Immunol 1993, 150:3459-3470.

80. Schulz O, Jaensson E, Persson EK, Liu X, Worbs T, Agace WW, Pabst O: Intestinal CD103+, but not CX3CR1+, antigen sampling cells migrate in lymph and serve classical dendritic cell functions. J Exp Med 2009, 206:3101-3114.

81. Denning TL, Norris BA, Medina-Contreras O, Manicassamy S, Geem D, Madan R, Karp CL, Pulendran B: Functional Specializations of Intestinal Dendritic Cell and Macrophage Subsets That Control Th17 and Regulatory T Cell Responses Are Dependent on the T Cell/APC Ratio, Source of Mouse Strain, and Regional Localization. J Immunol 2011.
82. Mullaly SC, Burrows K, Antignano F, Zaph C: Assessing the Role of CD103 in Immunity to an Intestinal Helminth Parasite. PLoS One 2011, 6:e19580.

83. Bochner BS: Siglec-8 on human eosinophils and mast cells, and Siglec-F on murine eosinophils, are functionally related inhibitory receptors. Clin Exp Allergy 2009, 39:317-324.

84. Marneros AG, Olsen BR: Physiological role of collagen XVIII and endostatin. FASEB J 2005, 19:716-728.

85. Van Itallie CM, Anderson JM: Claudins and epithelial paracellular transport. Annu Rev Physiol 2006, 68:403-429.

86. Rychter JW, Van Nassauw L, Brown JK, Van Marck E, Knight PA, Miller HR, Kroese $A B$, Timmermans JP: Impairment of intestinal barrier and secretory function as well as egg excretion during intestinal schistosomiasis occur independently of mouse mast cell protease-1. Parasite Immunol 2010, 32:221-231.

87. Metzger H: The receptor with high affinity for IgE. Immunol Rev 1992, 125:37-48.

88. Boros DL: Immunopathology of Schistosoma mansoni infection. Clin Microbiol Rev 1989, 2:250-269.

89. Lakhan SE, Kirchgessner A: Neuroinflammation in inflammatory bowel disease. J Neuroinflammation 2010, 7:37.

90. De Jonge F, Van Nassauw L, Van Meir F, Miller HR, Van Marck E, Timmermans JP. Temporal distribution of distinct mast cell phenotypes during intestinal schistosomiasis in mice. Parasite Immunol 2002, 24:225-231.

91. Andoh A, Deguchi Y, Inatomi O, Yagi Y, Bamba S, Tsujikawa T, Fujiyama Y: Immunohistochemical study of chymase-positive mast cells in inflammatory bowel disease. Oncol Rep 2006, 16:103-107.

92. Jankovic D, Kullberg MC, Dombrowicz D, Barbieri S, Caspar P, Wynn TA, Paul WE, Cheever AW, Kinet JP, Sher A: Fc epsilonRI-deficient mice infected with Schistosoma mansoni mount normal Th2-type responses while displaying enhanced liver pathology. J Immunol 1997, 159:1868-1875.

93. Yamamoto S, Isuzugawa K, Takahashi Y, Murase Y, Iwata M, Arisawa T, Nakano H, Nishimura N, Yamato S, Ohta M, Ina K, Murata T, Hori M, Ozaki H, Imakawa K: Intestinal gene expression in TNBS treated mice using genechip and subtractive cDNA analysis: implications for Crohn's disease. Biol Pharm Bull 2005, 28:2046-2053.

94. Saurer $\mathrm{L}$, Mueller $\mathrm{C}$ : T cell-mediated immunoregulation in the gastrointestinal tract. Allergy 2009, 64:505-519.

95. Petrof EO, Ciancio MJ, Chang EB: Role and regulation of intestinal epithelial heat shock proteins in health and disease. Chin J Dig Dis 2004, 5:45-50.

96. Reuter BK, Pizarro TT: Mechanisms of tight junction dysregulation in the SAMP1/YitFc model of Crohn's disease-like ileitis. Ann N Y Acad Sci 2009, 1165:301-307.

97. Gorfu G, Rivera-Nieves J, Ley K: Role of beta7 integrins in intestinal lymphocyte homing and retention. Curr Mol Med 2009, 9:836-850.

98. Strauch UG, Grunwald N, Obermeier F, Gurster S, Rath HC: Loss of CD103+ intestinal dendritic cells during colonic inflammation. World J Gastroenterol 2010, 16:21-29.

99. Cao C, Lawrence DA, Strickland DK, Zhang L: A specific role of integrin Mac-1 in accelerated macrophage efflux to the lymphatics. Blood 2005, 106:3234-3241.

100. Teller IC, Beaulieu JF: Interactions between laminin and epithelial cells in intestinal health and disease. Expert Rev Mol Med 2001, 3:1-18.

101. Bouatrouss Y, Herring-Gillam FE, Gosselin J, Poisson J, Beaulieu JF: Altered expression of laminins in Crohn's disease small intestinal mucosa. Am J Pathol 2000, 156:45-50

102. Kane CM, Jung E, Pearce EJ: Schistosoma mansoni egg antigen-mediated modulation of Toll-like receptor (TLR)-induced activation occurs independently of TLR2, TLR4, and MyD88. Infect Immun 2008, 76:5754-5759.

103. Katz J, Zhang P, Martin M, Vogel SN, Michalek SM: Toll-like receptor 2 is required for inflammatory responses to Francisella tularensis LVS. Infect Immun 2006, 74:2809-2816.

104. Melmed G, Thomas LS, Lee N, Tesfay SY, Lukasek K, Michelsen KS, Zhou Y, Hu B, Arditi M, Abreu MT: Human intestinal epithelial cells are broadly unresponsive to Toll-like receptor 2-dependent bacterial ligands: implications for host-microbial interactions in the gut. J Immuno/ 2003, 170:1406-1415.

105. Vijay-Kumar M, Wu H, Aitken J, Kolachala VL, Neish AS, Sitaraman SV, Gewirtz AT: Activation of toll-like receptor 3 protects against DSS-induced acute colitis. Inflamm Bowel Dis 2007, 13:856-864.

106. Wang KX, Denhardt DT: Osteopontin: role in immune regulation and stress responses. Cytokine Growth Factor Rev 2008, 19:333-345. 
107. Mercado-Lubo R, McCormick BA: The interaction of gut microbes with host ABC transporters. Gut Microbes 2010, 1:301-306.

108. Medina C, Radomski MW: Role of matrix metalloproteinases in intestinal inflammation. J Pharmacol Exp Ther 2006, 318:933-938.

109. Barrett JC, Hansoul S, Nicolae DL, Cho JH, Duerr RH, Rioux JD, Brant SR, Silverberg MS, Taylor KD, Barmada MM, Bitton A, Dassopoulos T, Datta LW, Green T, Griffiths AM, Kistner EO, Murtha MT, Regueiro MD, Rotter Jl, Schumm LP, Steinhart AH, Targan SR, Xavier RJ, Libioulle C, Sandor C, Lathrop M, Belaiche J, Dewit O, Gut I, Heath S, Laukens D, Mni M, Rutgeerts P, Van GA, Zelenika D, Franchimont D, Hugot JP, de VM, Vermeire S, Louis E, Cardon LR, Anderson CA, Drummond H, Nimmo E, Ahmad T, Prescott NJ, Onnie CM, Fisher SA, Marchini J, Ghori J, Bumpstead S, Gwilliam R, Tremelling M, Deloukas P, Mansfield J, Jewell D, Satsangi J, Mathew CG, Parkes M, Georges M, Daly MJ: Genome-wide association defines more than 30 distinct susceptibility loci for Crohn's disease. Nat Genet 2008, 40:955-962

110. Franke A, McGovern DP, Barrett JC, Wang K, Radford-Smith GL, Ahmad T, Lees CW, Balschun T, Lee J, Roberts R, Anderson CA, Bis JC, Bumpstead S, Ellinghaus D, Festen EM, Georges M, Green T, Haritunians T, Jostins L, Latiano A, Mathew CG, Montgomery GW, Prescott NJ, Raychaudhuri S, Rotter Jl, Schumm P, Sharma Y, Simms LA, Taylor KD, Whiteman D, Wijmenga C, Baldassano RN, Barclay M, Bayless TM, Brand S, Buning C, Cohen A, Colombel JF, Cottone M, Stronati L, Denson T, De VM, D'Inca R, Dubinsky M, Edwards C, Florin T, Franchimont D, Gearry R, Glas J, Van GA, Guthery SL, Halfvarson J, Verspaget HW, Hugot JP, Karban A, Laukens D, Lawrance I, Lemann M, Levine A, Libioulle C, Louis E, Mowat C, Newman W, Panes J, Phillips A, Proctor DD, Regueiro M, Russell R, Rutgeerts P, Sanderson J, Sans M, Seibold F, Steinhart AH, Stokkers PC, Torkvist L, Kullak-Ublick G, Wilson D, Walters T, Targan SR, Brant SR, Rioux JD, D'Amato M, Weersma RK, Kugathasan S, Griffiths AM, Mansfield JC, Vermeire S, Duerr RH, Silverberg MS, Satsangi J, Schreiber S, Cho JH, Annese V, Hakonarson H, Daly MJ, Parkes M: Genome-wide meta-analysis increases to 71 the number of confirmed Crohn's disease susceptibility loci. Nat Genet 2010, 42:1118-1125.

111. Lees CW, Barrett JC, Parkes M, Satsangi J: New IBD genetics: common pathways with other diseases. Gut 2011.

112. McGovern DP, Gardet A, Torkvist L, Goyette P, Essers J, Taylor KD, Neale BM, Ong RT, Lagace C, Li C, Green T, Stevens CR, Beauchamp C, Fleshner PR, Carlson M, D'Amato M, Halfvarson J, Hibberd ML, Lordal M, Padyukov L, Andriulli A, Colombo E, Latiano A, Palmieri O, Bernard EJ, Deslandres C, Hommes DW, de Jong DJ, Stokkers PC, Weersma RK, Sharma Y, Silverberg MS, Cho JH, Wu J, Roeder K, Brant SR, Schumm LP, Duerr RH, Dubinsky MC, Glazer NL, Haritunians T, Ippoliti A, Melmed GY, Siscovick DS, Vasiliauskas EA, Targan SR, Annese V, Wijmenga C, Pettersson S, Rotter JI, Xavier RJ, Daly MJ, Rioux JD, Seielstad M: Genome-wide association identifies multiple ulcerative colitis susceptibility loci. Nat Genet 2010, 42:332-337.

doi:10.1186/1471-2164-13-377

Cite this article as: Avula et al.: Whole-genome microarray analysis and functional characterization reveal distinct gene expression profiles and patterns in two mouse models of ileal inflammation. BMC Genomics 2012 13:377.

\section{Submit your next manuscript to BioMed Central and take full advantage of:}

- Convenient online submission

- Thorough peer review

- No space constraints or color figure charges

- Immediate publication on acceptance

- Inclusion in PubMed, CAS, Scopus and Google Scholar

- Research which is freely available for redistribution 